\title{
The Novel LSD1 Inhibitor ZY0511 Suppresses Diffuse Large B Cell Lymphoma Proliferation by Inducing Apoptosis and Autophagy
}

Huan Liu

Sichuan University

Jing Wei

Sichuan University

$\mathrm{Na}$ Sang

Sichuan University

Xi Zhong

Sichuan University

Xia Zhou

Sichuan University

Xinyu Yang

Sichuan University

Jing Zhang

Sichuan University

Zeping Zuo

Sichuan University

Yang Zhou

Sichuan University

Shengyong Yang

Sichuan University

Junrong Du

Sichuan University

Yinglan Zhao ( $\nabla$ zhaoyinglan@scu.edu.cn )

Sichuan University https://orcid.org/0000-0002-5168-5842

\section{Research Article}

Keywords: LSD1 inhibitor, ZY0511, diffuse large B cell lymphoma, apoptosis, autophagy

Posted Date: June 3rd, 2021

DOI: https://doi.org/10.21203/rs.3.rs-578853/v1 
License: (c) (i) This work is licensed under a Creative Commons Attribution 4.0 International License. Read Full License 


\section{Abstract}

Lysine-specific demethylase 1 (LSD1, also known as KDM1A) is an attractive agent for treatment of cancer. Nevertheless, the anti-tumor effect of LSD1 inhibitors against diffuse large B-cell lymphoma (DLBCL) and the underlying mechanism are still unclear. Here, we report that $K D M 1 A$ is overexpressed in human DLBCL tissues, and negatively related to overall survival rate in DLBCL patients. ZY0511, a novel and potent LSD1 inhibitor, developed by our group inhibited the proliferation of human DLBCL cells and blocked cell cycle at G0/G1 phase. Cellular thermal shift assay (CETSA) showed that ZY0511 interacted with LSD1 in DLBCL cells. Furthermore, intraperitoneal administration of ZY0511 significantly suppressed SU-DHL-6 xenograft tumor growth in vivo. Mechanistically, transcriptome sequencing results indicated that ZY0511 induced the genes enrichment significantly related to cell cycle, autophagy and apoptosis signaling pathways. Further study confirmed that ZY0511 decreased mitochondrial membrane potential and induced apoptosis, which can be reverted by a pan-caspase inhibitor, Z-VAD-FMK. Moreover, ZY0511 treatment significantly increased autophagy-associated marker proteins and autophagosomes formation in DLBCL cells. In conclusion, our finding identifies that ZY0511 inhibits DLBCL growth both in vitro and in vivo via the induction of apoptosis and autophagy, and LSD1 inhibitor might be a promising strategy for treating DLBCL.

\section{Introduction}

Diffuse large B cell lymphoma (DLBCL) is the most aggressive class of non-Hodgkin lymphomas (NHLs) [1]. The standard treatment strategy of rituximab in combination with a cocktail of chemotherapy agents comprising of cyclophosphamide, doxorubicin, prednisone and vincristine (R-CHOP) can extend the overall survival time of DLBCL patients. However, there are still $40 \%$ of patients who do not have response or relapse after treatment [2], which made the outcome of DLBCL patients remains far from satisfactory. Therefore, it is urgent to develop novel drugs that can be effectively used for treatment of DLBCL patients.

Epigenetic dysregulation plays a crucial role in the initiation and development in human DLBCL $[1,3]$. In 2004, Shi's group identified that lysine-specific histone demethylase 1 (LSD1, also known as AOF2, BC110 and KDM1A) was an epigenetic regulatory factor [4]. LSD1 represses transcription via specially demethylating lysine residues of histone H3K4 and activates transcription though specially demethylating lysine residues of histone $\mathrm{H} 3 \mathrm{~K} 9$, respectively, thereby regulating the expression of target genes [5]. LSD1 is ubiquitously overexpressed in numerous cancers, including acute myeloid leukemia (AML), breast cancer, prostate cancer, neuroblastoma, and small cell lung cancer (SCLC), and its overexpression associates with the initiation and progression of malignant tumors and the overall survival time of patients. Therefore, LSD1 has attached much attention, and the biological function of LSD1 in hematological malignancies especially AML has been extensive studied. In AML, LSD1 interacts with ectopic SNAI1 to induce myeloid development defects [6]. Furthermore, there are several studies suggested that LSD1 participates in lymphomagenesis and the progression of lymphoma. LSD1 is found to be essential for germinal centers (GC) formation and humoral immune response by interacting with 
BCL6 in lymphoma cells. Conditional deletion of LSD1 obviously delayed BCL6-driven lymphomagenesis [7]. Above studies revealed that LSD1 play a crucial role in lymphoma, suggesting that it is feasible to treat DLBCL with LSD1 inhibitors.

In the last decades, numerous LSD1 inhibitors, including TCP, ORY-1001, ORY-2001, CC-90011, INCB059872 and IMG-7289 are undergoing clinical trials for treatment of AML, myelodysplastic syndromes, myeloproliferative neoplasms, relapsed/refractory NHL, SCLC, relapsed Ewing sarcoma, sickle cell disease, myelofibrosis, essential thrombocythemia, multiple sclerosis, mild to moderate Alzheimer's disease and solid tumors [8]. However, the effect of LSD1 inhibitors in DLBCL treatment remains largely unclear.

In 2016, our group developed a potent LSD1 inhibitor, ZY0511, which exhibited anti-cancer activity against numerous human cancer cell lines [9]. Here, we aimed to clarify the anti-tumor effect and underlying mechanism of ZY0511 against DLBCL. We found that KDM1A was highly expressed in human DLBCL tissues and associated with the poor survival of DLBCL patients. By interacting with LSD1 in DCBCL cells, ZY0511 inhibited cell proliferation both in vitro and in vivo. Mechanistically, ZY0511 blocked cell cycle at G0/G1 phase, induced both the endogenous and exogenous apoptosis, and induced autophagy via inactivation of mTOR/p70S6K signaling pathways. Our findings reveal that ZY0511 might be a novel and promising treatment strategy for treating DLBCL.

\section{Materials And Methods}

\section{Antibodies and reagents}

The main antibody information was listed below: LSD1 (CST, cat\# 2139), H3 (CST, cat\# 4499), H3K4me (CST, cat\# 5326), H3K4me2 (CST, cat\# 9725), H3K9me (CST, cat\# 14186), H3K9me2 (CST, cat\# 4658), phospho-p53 (Ser 15,CST, cat\# 9284), phospho-mTOR (Ser 2448, CST, cat\# 5536), p70 S6K (CST, cat\# 2708), phospho-p70 S6k (Thr 421/Ser 424, CST, cat\# 9204), phospho-S6 (CST, Ser235/236, cat\#2211), Beclin-1 (CST, cat\# 3495), Bax (CST, cat\# 2772), Cleaved Caspase-3 (CST, cat\# 9664), GAPDH (CST, cat\# 2118), Ki67 (Abcam, cat\# ab16667), mTOR (ZEN BIO, cat\# 380411), p53 (Proteintech, cat\# 10442-1-AP), Caspase-8 (Proteintech, cat\# 13434-1-AP), CDK4 (Proteintech, cat\# 11026-1-AP), CDK6 (Proteintech, cat\# 14052-1-AP), Cyclin D1 (Proteintech, cat\# 60186-1-AP), LC3B (Santa Cruz, sc-376404), p62/SQSTM1 (HUA BIO, cat\# R1309-8), S6 (Abclonel, cat\# A6058), PCNA (Gservice, Gb13030-2).

GSK2879552, 3MA, chloroquine (CQ) and Z-VAD-FAM were purchased from Selleckchem (TX, USA). ZY0511 was synthesized in laboratory as described previously [9].

\section{Cell Lines and cell culture}

The human DLBCL cell lines including SU-DHL-4, SU-DHL-6, SU-DHL-10 and Farage were purchased from American Type Culture Collection (ATCC, VA, USA). The DLBCL cells were cultured in RPMI 1640 medium 
containing $20 \%$ fetal bovine serum (Gibco), at $37^{\circ} \mathrm{C}$ and $5 \% \mathrm{CO}_{2}$.

\section{Western blot}

The human DLBCL cells were lysed with RIPA lysis buffer containing protease inhibitor and phosphatase inhibitors. Next, the BCA (bicinchoninic acid) method was used to quantify the protein concentrations. Proteins were isolated by SDS-PAGE gel, and then transferred onto polyvinylidene fluoride membranes (Millipore). After blocking with 5\% skimmed milk, the membranes were incubated with the specific antibodies at $4^{\circ} \mathrm{C}$ overnight. Washed the membranes with TBST buffer three times and incubated with HRP conjugated secondary antibodies at room temperature for $1 \mathrm{~h}$. At last, the protein levels were visualized using Chemiluminescent HRP Substrate (Millipore) and signals were detected using chemiluminescence imaging system.

\section{Cellular thermal shift assay (CETSA)}

The DLBCL cells were seeded $\left(2 \times 10^{5}\right.$ cells $\left./ \mathrm{mL}\right)$ and treated with DMSO or ZY0511 at the final concentration of $200 \mu \mathrm{M}$ at $37^{\circ} \mathrm{C}$ for $1 \mathrm{~h}$. The cells were harvested and then washed with pre-chilled PBS for twice, followed by resuspension in pre-chilled PBS containing protease inhibitor. The cell suspension were aliquoted into PCR tubes $\left(1.5 \times 10^{6}\right.$ cells/tube) and heated at different temperatures $\left(42^{\circ} \mathrm{C}, 44^{\circ} \mathrm{C}\right.$, $46^{\circ} \mathrm{C}, 48^{\circ} \mathrm{C}, 50^{\circ} \mathrm{C}, 52^{\circ} \mathrm{C}, 54^{\circ} \mathrm{C}$ ) for $3 \mathrm{~min}$, followed by placed at room temperature for $3 \mathrm{~min}$. Next, the sample was subjected to three freeze (in liquid nitrogen) - thaw cycles (at room temperature), and the supernatant was obtained by centrifugation at $20000 \times \mathrm{g}$ for $20 \mathrm{~min}$ at $4^{\circ} \mathrm{C}$. Subsequently, loading buffer was added, and then proteins were denatured at $100^{\circ} \mathrm{C}$ for $10 \mathrm{~min}$. Finally, the level of LSD1 was detected by western blot assay. Western blot signals based on densitometry method were quantified by Fiji and CETSA curves in intact cells were graphed by GraphPad Prism 8 software (San Diego, USA).

\section{Cell proliferation inhibition determined by the AO/PI (Acridine Orange/ Propidium lodide) method}

The DLBCL cells were seeded $\left(2 \times 10^{5}\right.$ cells $\left./ \mathrm{mL}\right)$ and treated with ZY0511 $(2 \mu \mathrm{M})$ and/or Z-VAD-FMK (50 $\mu \mathrm{M})$ for $48 \mathrm{~h}$. The cell number and viability were determined by an AO/PI exclusion test. The results were plotted as means \pm SEM of three separate experiments.

\section{MTT assay}

An MTT (Sigma, USA) experiment was conducted to assess the proliferation inhibitory rate of ZY0511 against DLBCL cells. The DLBCL cells were treated with various concentrations of ZY0511 in 96-well plates for $24 \mathrm{~h}\left(8 \times 10^{4}\right.$ cells/well), $48 \mathrm{~h}\left(4 \times 10^{4}\right.$ cells/well), $72 \mathrm{~h}\left(2 \times 10^{4}\right.$ cells/well $)$ and $96 \mathrm{~h}\left(1 \times 10^{4}\right.$ 
cells/well), respectively. Then, $20 \mu \mathrm{L}$ MTT $(5 \mathrm{mg} / \mathrm{mL})$ was added to form formazan. Optical density was measured using a microplate reader (Thermo Fisher Scientific, USA). The IC $\mathrm{C}_{50}$ values were calculated by GraphPad Prism 8 software using XY modeling.

\section{EdU incorporation assay}

The DLBCL cells were seeded $\left(2 \times 10^{5}\right.$ cells $\left./ \mathrm{mL}\right)$ and treated with ZY0511 at the final concentrations of $0.5,1,2 \mu \mathrm{M}$ for $24 \mathrm{~h}$. The DLBCL cells were grown in medium containing EdU, which is a thymidine analogue labelled cells in the proliferation phase for $2 \mathrm{~h}$. Then, the EdU positive cells were stained with Cell-Light EdU Apollo488 In Vitro Flow Cytometry Kit according to Ribobo's instructions (Shanghai, China). Cells were measured by flow cytometry (FCM) (Agilent NovoCyte, San Diego, USA) and analyzed using NovoExpress software (Agilent NovoCyte, San Diego, USA).

\section{Cell cycle analysis}

The DLBCL cells were seeded $\left(2 \times 10^{5}\right.$ cells $\left./ \mathrm{mL}\right)$ and treated with ZY0511 at the final concentrations of $0.5,1,2 \mu \mathrm{M}$ for $24 \mathrm{~h}$. Then, the DLBCL cells were collected and fixed with $70 \%$ ethanol solution at $4^{\circ} \mathrm{C}$ overnight. Fixed cells were stained with propidium iodide according to KeyGEN BioTECH's instructions (Jiangsu, China). Cells were measured by FCM (Agilent NovoCyte, San Diego, USA) and analyzed using NovoExpress software (Agilent NovoCyte, San Diego, USA).

\section{Apoptosis analysis}

The DLBCL cells were seeded $\left(2 \times 10^{5}\right.$ cells $\left./ \mathrm{mL}\right)$ and treated with ZY0511 at the final concentrations of $0.5,1,2 \mu \mathrm{M}$ for $48 \mathrm{~h}$. Then, the DLBCL cells were harvested and stained with Annexin V-PE and 7-AAD according to BD's instructions (San Diego, USA), followed by apoptosis analysis by FCM [10].

\section{Mitochondrial membrane potential $(\Delta \Psi \mathrm{m})$ assay}

The DLBCL cells were seeded $\left(2 \times 10^{5}\right.$ cells $\left./ \mathrm{mL}\right)$ and treated with ZY0511 at the final concentrations of $0.5,1,2 \mu \mathrm{M}$ for $48 \mathrm{~h}$. Then, the DLBCL cells were harvested and incubated with tetraethyl benzimidazolyl carbocyanine iodide (JC-1) according to Beyotime's instructions (Shanghai, China), followed by $\Delta \Psi \mathrm{m}$ assay by FCM.

\section{Real-Time Quantitative PCR (qRT-PCR)}

Total RNA was isolated from cultivated DLBCL cells using AxyPrep ${ }^{T M}$ Multisource Total RNA Miniprep Kit (Axygen, USA). PrimeScript ${ }^{\mathrm{TM}}$ RT reagent Kit with Gdna Eraser (TakaRa, Japan) was used to synthesize 
cDNA. RNA quality was evaluated by Real-Time system (Bio-Rad, USA) using the SYBR ${ }^{\circledR}$ Green Supermix (Bio-Rad, USA). Primers were listed in Table 1.

Table 1.

Primers

\begin{tabular}{|lll|}
\hline Target & Forward primer & Reverse primer \\
\hline GAPDH & CAGGAGGCATTGCTGATGAT & GAAGGCTGGGGCTCATTT \\
\hline MYC & ATGCCCCTCAACGTTAGCTT & CTCCTCCTCGTCGCAGTAGA \\
\hline PCNA & CCTGCTGGGATATTAGCTCCA & CAGCGGTAGGTGTCGAAGC \\
\hline CDKN1A & AGGGGACAGCAGAGGAAGAC & GCCGTTTTCGACCCTGAGAG \\
\hline ATM & TGCTTATCTGCTGCCGTCAA & TCAGGATCTCGAATCAGGCG \\
\hline CDK4 & GTGTACAAGGCCCGTGATCC & GTCGCCTCAGTAAAGCCACC \\
\hline CDK6 & CAGGTGGCCCTCGGAATAGA & ACATGACACCTACGAGGGCA \\
\hline CCND1 & GCCCTCGGTGTCCTACTTCAAATG & TCCTCCTCGCACTTCTGTTCCTC \\
\hline FAS & TCTGGTTCTTACGTCTGTTGC & CTGTGCAGTCCCTAGCTTTCC \\
\hline mTOR & GCACGTCAGCACCATCAACCTC & CTCAGCCATTCCAGCCAGTCATC \\
\hline BNIP3 & CAGCGTTCCAGCCTCGGTTTC & AGCTACTCCGTCCAGACTCATGC \\
\hline ULK1 & CTGCCTGTCGTCCACTGTGAAG & CCGCTGCTTGTCCAGGAAGAAG \\
\hline ATG9A & AGCAGGTTCAGCGGGATGGAG & ACCACATTTGCGATAAGGCTCAGG \\
\hline SQSTM1 & TGATTGAGTCCCTCTCCCAGATGC & CCGCTCCGATGTCATAGTTCTTGG \\
\hline ATG7 & GAGCAAGCCCGCAGAGATGTG & TCCCAAAGCAGCATTGATGACCAG \\
\hline ATG3 & AGGCATACCTACCAACAGGCAAAC & CCATCCGCCATCACCATCATCTTC \\
\hline MAP1LC3B & GCAGGGTAAAGGGCTGTG & GAGTGAGGACTTTGGGTTGGTTC \\
\hline BECN1 & CCATGCAGGTGAGCTTCGT & GAATCTGCGAGAGACACCATC \\
\hline
\end{tabular}

\section{RNA Sequencing (RNA-seq)}

RNA-seq experiment was conducted using a profiler service provided by Novogene Co. Ltd (Beijing, China). After $48 \mathrm{~h}$ of treatment with DMSO or ZY0511 $(1 \mu \mathrm{M})$, total RNA was purified from SU-DHL-4 cells and stored in TRIzol (Invitrogen, USA). Use a fold-change $>2.0$, FDR (false discovery rate) $<0.05$ and $P<$ 
0.05 (Student's t-test) to filter significant probe sets for each biological sample $(n=3)$. Calculate the gene counts of each transcript and use DESeq2 for normalization to determine differentially expressed genes.

\section{Immunofluorescence (IF)}

After ZY0511 treatment, DLBCL cells were harvested and fixed with $4 \%$ paraformaldehyde at $4^{\circ} \mathrm{C}$ for 15 min. The cell suspension was dripped onto a glass slide and placed at $37^{\circ} \mathrm{C}$ to dry the water. At room temperature, Permeabilize the DLBCL cells with $0.2 \%$ Triton X-100 solution for 20 min and block the permeabilized cells with PBST containing $0.2 \%$ BSA for $1 \mathrm{~h}$. Then, incubate the slides with primary antibody overnight at $4^{\circ} \mathrm{C}$, then incubate with cy3 or FITC-conjugated secondary antibody for $1 \mathrm{~h}$ at room temperature with shaking. Nuclei were labeled with DAPI. DeltaVision Ultra-high resolution microscope (GE Healthcare, USA) was used to analyze the slides using a $63 \times$ NA oil objective.

\section{Transmission electron microscopy (TEM)}

The DLBCL cells were seeded $\left(2 \times 10^{5}\right.$ cells $\left./ \mathrm{mL}\right)$ and treated with ZY0511 at the final concentration of 2 $\mu \mathrm{M}$ for $48 \mathrm{~h}$. The treated cells were fixed with $2.5 \%$ glutaraldehyde. Then, cells were embedded in epoxy resin and imaged by TEM (JEM-1400 Plus, Japan) following standard TEM procedures.

\section{Subcutaneous xenograft models}

NOD/SCID mice aged five- to six-week were purchased from the GemPharmatech Co., Ltd (Jiangsu, China) and fed under specific pathogen-free (SPF) barriers. The SU-DHL-6 Cells $\left(2 \times 10^{6}\right.$ cells $/ \mathrm{mouse} / 100$ $\mu \mathrm{L}$ ) were subcutaneously injected in the right flank of the mice. The tumor-bearing mice were randomly divided into three groups ( $\mathrm{n}=6$ per group) when tumor volume reached $80-100 \mathrm{~mm}^{3}$. The ZY0511 (50 $\mathrm{mg} / \mathrm{kg}$ and $100 \mathrm{mg} / \mathrm{kg}$ ) or solvent were administrated once daily by intraperitoneal injection for 21 days. Tumor volumes and body weights were monitored every three days. Calculation formula: $\mathrm{V}\left(\mathrm{mm}^{3}\right)=(\mathrm{a} \times$ $\left.b^{2}\right) / 2$ ( $V$ is the tumor volume, $a$ is the length and $b$ is the width). At the end of the experiment on day 21 , the tumor tissues were stripped and fixed in $4 \%$ paraformaldehyde for further experiments.

\section{Immunohistochemistry analysis and H\&E staining}

After treating with ZY0511 for 21 days, tumor tissues were collected from mice bearing SU-DHL-6 tumors. After being fixed in $4 \%$ paraformaldehyde for $48 \mathrm{~h}$, the tumor tissues were embedded in paraffin, and then stained with Ki67 and PCNA by immunohistochemistry analysis. H\&E staining tissue samples were the same as above.

\section{Statistical analysis}


Data are presented as the means \pm SEM. GraphPad Prism 8 software was used to perform statistical analyses. The statistical significance of the data between two experimental groups was detected by a two-tailed Student's t test. The statistical significance of the data among multiple groups was tested with one-way ANOVA. The survival analysis was performed by the log-rank test. $P<0.05$ were considered statistically significant.

\section{Results}

\section{$K D M 1 A$ is overexpressed in human DLBCL tissues and negatively related to overall survival of patients}

LSD1 is highly expressed in multiple human cancers. To further explore the clinical significance and correlation of LSD1 in DLBCL patients, we used a database containing 384 individual DLBCL tissues from the GEPIA2 website (http://gepia2.cancer-pku.cn/\#index) to analyze the expression of KDM1A mRNA in human DLBCL tissues and normal lymph nodes. The results demonstrated that high expression of KDM1A was observed in DLBCL tissues (Fig. 1a). We carried out further analysis on the Genomicscape website (http://www.genomicscape.com/). It was found that the KDM1A low expression group (KDM1Alow) had a higher survival rate than the KDM1A high expression group (KDM1A-high). Moreover, high $K D M 1 A$ expression in DLBCL cancer tissues was associated with poor prognosis of patients by KaplanMeier survival analysis (Fig. 1b). In short, these data indicate that the high expression of KDM1A in human DLBCL tissues is tightly corelated with the initiation and progression of DLBCL.

\section{ZY0511 interacts with LSD1 in DLBCL cells}

The cellular thermal shift assay (CETSA), the first widely applicable biophysical technique, was used to investigate the binding of ligands to proteins in intact cells [11]. After drug binding, the protein usually becomes structurally stable and its thermal stability is improved [11]. Therefore, we performed CETSA to evaluate whether ZY0511 interacted with LSD1 in DLBCL cells. The results revealed ZY0511 $(100 \mu \mathrm{M})$ treatment induced large thermal shifts of LSD1 in DLBCL cells compared with control. ZY0511 treatment increased the melt temperature of LSD1 in SU-DHL-4, SU-DHL-6, SU-DHL-10, and Farage cells 2.04, 1.27, $0.87,1.72^{\circ} \mathrm{C}$, respectively (Fig. $1 \mathrm{C}-\mathrm{f}$ ). These results suggested that ZY0511 interacted with LSD1 and thus increased the thermal stability of LSD1 in DLBCL cells, which is a crucial factor in the treatment effect.

To further confirm ZY0511 interacted with LSD1 and affected its catalytic function in DLBCL cells, we detected the histone methylation level as LSD1 is a histone-modifying enzyme that demethylates lysine residues of histone $\mathrm{H} 3 \mathrm{~K} 4$ and $\mathrm{H} 3 \mathrm{~K} 9$ [4]. The result demonstrated that ZY0511 significantly increased the H3K4me and H3K9me2 levels (Fig. 1g). Simultaneously, ZY0511 had no influence on expression of LSD1 (Fig. 1f). Taken together, ZY0511 interacted with LSD1 in DLBCL cells, conforming that ZY0511 targeted LSD1 and exhibited catalytic function. 


\section{ZY0511 inhibits cell proliferation and induces cell cycle arrest at G0/G1 phase in vitro}

To study the potential functional roles of ZY0511 in DLBCL cells, the cells proliferation after ZY0511 treatment was investigated by the MTT assay. The results revealed that ZY0511 time-dependently displayed considerable anti-viability activities against SU-DHL-4, SU-DHL-6, SU-DHL-10, and Farage cells with $\mathrm{IC}_{50}$ values ranging from 0.3-2.8 $\mu \mathrm{M}$ (Fig. 2a). SU-DHL-6 cells was the most sensitive cell line to ZY0511, with $\mathrm{IC}_{50}$ values $1.08 \mu \mathrm{M}$ for $24 \mathrm{~h}, 0.66 \mu \mathrm{M}$ for $48 \mathrm{~h}, 0.48 \mu \mathrm{M}$ for $72 \mathrm{~h}$, and $0.39 \mu \mathrm{M}$ for $96 \mathrm{~h}$, respectively. Response to ZY0511, the sensitivity of SU-DHL-4 cells is second only to that of SU-DHL-6 cells. We next used SU-DHL-4 cells and SU-DHL-6 cells for further investigation. EdU incorporation assay further confirmed that after ZY0511 treatment, the DNA synthesis in DLBCL cells was obviously decreased in a concentration-dependent manner, which is same as cell proliferation inhibition induced by ZY0511 (Fig. 2b).

The effect of ZY0511 on the cell cycle was assessed in above cell lines. Experimental results revealed that ZY0511 concentration-dependently induced cell cycle arrest at G0/G1 phase (Fig. 2c-d). As expected, immunoblot analyses found the levels of p53 and p-P53 was elevated, CDK4 and Cyclin D1 was reduced, whereas the expression of CDK6 didn't change significantly (Fig. 2e). The above data indicated that ZY0511 inhibits DLBCL cells proliferation and induces G0/G1 cell cycle phase arrest in vitro.

\section{ZY0511 inhibits tumor growth in vivo}

The above studies confirmed the in vitro anti-tumor activity of ZY0511. Next, we evaluated the in vivo anti-tumor efficacy of ZY0511 using subcutaneous xenograft models of SU-DHL-6 cells, which was the most sensitive cell line to ZY0511. NOD/SCID mice were injected intraperitoneally with ZY0511 daily for 21 days. The results showed that ZY0511 resulted in significant tumor growth inhibition with a 46\% tumor-inhibitory rate at the dose of $50 \mathrm{mg} / \mathrm{kg}$, and a $68 \%$ tumor-inhibitory rate at the dose of $100 \mathrm{mg} / \mathrm{kg}$ (Fig. 3a-c). Notably, ZY0511 didn't result in significant body weight loss of mice (Fig. 3d). Consistent with the in vitro data, the Ki67 and PCNA levels in tumor tissues were markedly decreased, indicating ZY0511 treatment inhibited the growth of tumor cells in vivo (Fig. 3e).

\section{ZY0511 results gene expression changes in cell cycle, apoptosis and autophagy pathways}

All above results confirmed that ZY0511 has anti-tumor effects in vitro and in vivo. We performed RNAseq analysis to comprehensively explore the anti-tumor mechanism mediated by ZY0511. The results showed that LSD1 inhibition obviously disturbed gene expression of SU-DHL-4 cells (Fig. 4a). Gene ontology (GO) enrichment analysis of the differentially expressed genes revealed enrichment for transcriptional programs significantly related to cell cycle, autophagy and apoptosis signaling pathways 
(Fig. 4b), which were further confirmed by KEGG pathway analysis (Fig. 4c). Among cell cycle-related genes, ZY0511 up-regulated CDKN1A expression by 5.6 folds and down-regulated $C D K 4$ expression by $60 \%$. Among apoptosis and autophagy-related genes, ZY0511 increased BNIP3 expression by 7.3 folds, ULK 1 expression by 3.6 folds, ATG9A expression by 2.0 folds, SQSTM1 expression by 1.9 folds, MAP1LC3B expression by 2.0 folds and decreased $m T O R$ expression by $50 \%$, ATG3 expression by $60 \%$, ATG7 expression by $10 \%$. Using LSD1 downstream gene sets (http://cistrome.org/CistromeCancer/) for gene set enrichment analysis (GSEA), it was found that ZY0511 treatment induced the change of a subset of LSD1 downstream transcriptional programs in SU-DHL-4 (Fig. 4d), suggesting that ZY0511 changes numerous genes regulated by LSD1.

We next performed qRT-PCR analysis to verify above-mentioned representative differential genes selected from RNA-seq (Fig. 4e). Validation results were consistent with RNA-seq findings. Collectively, the antitumor mechanism of ZY0511 may be association with cell cycle arrest, apoptosis and autophagy pathways.

\section{ZY0511 exhibits anti-tumor effects by inducing apoptosis}

To study whether ZY0511 induced apoptosis in DLBCL, we performed FCM analysis to detect apoptosis cells using Annexin V/7AAD staining. Results indicated that apoptosis was concentration-dependently induced in ZY0511 group compared with the control (Fig. 5a-b). Western blot analysis of cleavage of caspase-3 (CC3), the indicator of cellular apoptosis, further supported this finding. Results showed that ZY0511 triggered extrinsic apoptosis pathway via cleavage of caspase-8 (Fig. 5e). Bcl-2 family proteins are essential for regulating the integrity of mitochondria. The level of Bcl-2-associated X protein[12] (Bax), a pro-apoptotic protein, was significantly increased (Fig. 5e), which implied that the mitochondrialmediated endogenous apoptosis might induced by ZY0511. Loss of mitochondrial membrane potential [13] $(\Delta \Psi \mathrm{m})$ was also associated with intrinsic apoptosis. After JC-1 staining, the depolarized cells, which represents the $\triangle \Psi \mathrm{m}$ alteration, was detected using FCM. As shown in Fig. 5c-d, the depolarized cells of all four DLBCL cell lines elevated dramatically as the concentration of ZY0511 increased, suggesting that the $\Delta \Psi \mathrm{m}$ levels decreased extremely after ZY0511 treatment. These findings suggested that ZY0511 induces both endogenous and exogenous apoptosis.

Then, to determine whether apoptosis is important for DLBCL cells death, we added Z-VAD-FMK, a pancaspase inhibitor, to the culture medium to culture cells. Next, to investigate the influence of Z-VAD-FAM on the ZY0511-induced cell death, we performed FCM analysis to detect apoptosis cells using Annexin V/7AAD staining. ZY0511 increased the number of apoptotic SU-DHL-4 cells by 7.9 folds (from 6.74 to $53.13 \%$ ), and Z-VAD-FMK cotreatment reduced apoptotic SU-DHL-4 cells by about $70 \%$ (from 53.13 to 15.72\%). ZY0511 increased the number of apoptotic SU-DHL-6 cells by 12.4 folds (from 6.62 to $81.78 \%$ ), and Z-VAD-FMK cotreatment reduced apoptotic SU-DHL- 6 cells by about $49 \%$ (from 81.78 to $41.8 \%$ ) (Fig. $5 f-g)$. Western blot results further indicated that ZY0511 alone obviously increased the expression of CC3, but this effect was completely reverted by ZVAD-FMK (Fig. 5h). Moreover, the effect of ZVAD-FMK on 
ZY0511 induced cell number and viability change were evaluated by AO/PI method. In SU-DHL-4 cells, ZVAD-FMK cotreatment increased the number of live cells by about $56 \%$ (from $1.60 \times 10^{5}$ to $2.51 \times 10^{5}$ cells/mL) compared with ZY0511 group. In SU-DHL-6 cells, Z-VAD-FMK cotreatment increased the number of live cells by about $53 \%$ (from $1.13 \times 10^{5}$ to $1.73 \times 10^{5}$ cells $/ \mathrm{mL}$ ) compared with ZY0511 group (Fig. 5i). Compared with ZY0511 group, Z-VAD-FMK cotreatment elevated the cell viability from $66 \%$ to $95 \%$ in SU-DHL-4 cells and from $43 \%$ to $62 \%$ in SU-DHL-6 cells (Fig. 5j). These findings demonstrated that the cell viability decreased by ZY0511 treatment was attenuated after ZVAD-FMK treatment. Thus, Z-VADFAM likely counteracts ZY0511-induced apoptosis by increasing resistance to apoptotic death. Therefore, apoptosis may be the important cause involved in DLBCL cells death induced by ZY0511.

\section{ZY0511 exhibits anti-tumor effects by inducing autophagy}

Cell death is mediated by multiple mechanisms such as apoptosis, autophagy cell death and necrosis. To ascertain whether ZY0511 induced autophagy in DLBCL cells, we first detected the levels of LC3B, p62 and Beclin-1, the markers of autophagy. After $24 \mathrm{~h}$ treatment, ZY0511 significantly upregulated the expression of p62 and meanwhile strongly induced LC3-I converting into LC3-II, while the level of Beclin-1 remained unchanged (Fig. 6a). This result was further confirmed by immunofluorescence staining. The levels of LC3B and p62 were obviously higher in ZY0511 group than in the control group (Fig. 6b-C). Moreover, TEM revealed swelling mitochondria and more autophagosomes in the ZY0511-treated group (Fig. 6d). To further proof that autophagy is specifically induced by ZY0511, $2.5 \mathrm{mM} 3 \mathrm{MA}$ or $10 \mu \mathrm{M}$ chloroquine (CQ) , autophagy inhibitors, was added into the culture media with ZY0511 [14]. As shown in Fig. 6e, 3MA can block the upstream of autophagy, and when combined with ZY0511, it can reduce the ratio of LC3-囚/LC3-囚. CQ can block the downstream of autophagy, and when combined with ZY0511, it

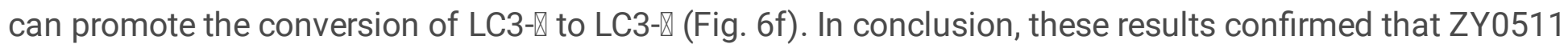
triggers excessive autophagy.

It has been well known that the mTOR/p70S6K signaling pathway play a crucial role in regulating autophagy [15]. In order to clarify how ZY0511 induced autophagy, the effects of ZY0511 on this signaling pathway were studied. We found that ZY0511 reduces phosphorylation of mTOR, p70 S6 Kinase (p70S6K) and ribosomal protein S6 (S6) in a concentration-dependent manner (Fig. 6g), confirming that autophagy induced by ZY0511 was at least in part mediated by the mTOR/p70S6K signaling pathway.

\section{Discussion}

As a genetically heterogeneous tumors, the clinical outcome of DLBCLs remains far from satisfactory within current clinical treatment. It's urgently needed to develop novel treatment strategies to improve the therapeutic outcomes of DLBCL. Thus, we investigated the anti-tumor effect of ZY0511 against DLBCL cells and the underlying mechanisms. ZY0511 induced the proliferation inhibition of DLBCL cells and G0/G1 cell cycle arrest. Consistent with in vitro data, ZY0511 significantly suppressed SU-DHL-6 
xenograft tumor growth in vivo. We further revealed that inhibition of LSD1 increased level of apoptosis and autophagy, which synergistically resulted the death of DLBCL cells. Collectively, our study provides a LSD1 inhibitor based novel strategy for the clinical treatment of DLBCL.

Recently, researchers have increasingly studied the anti-tumor effect and mechanism of epigenetic inhibitors against DLBCL. It was found that pharmacologically inhibition of CREBBP/EP300, histone acetyltransferase, resulted synthetic lethality of DLBCL cells [16]. Tazemetostat, an EZH2 inhibitor, is entered phase II clinical trial for NHL treatment, including DLBCL [17]. LSD1, an epigenetic regulatory factor, plays a crucial role in the initiation and development of various tumors. The high expression of LSD1 protein has been observed in various tumors and pharmacological inhibition of LSD1 by inhibitors such as GSK2879552 and IMG-7289 or LSD1 knockdown by RNA interference exhibited potent anticancer efficiency. We found that KDM1A is significantly increased in human DLBCL tissues compared with normal tissues, and is negatively corrected with overall survival of DLBCL patients. This is not surprising as the elevated expression of LSD1 were found in various hematological malignancies such as AML. This result aroused our interesting that LSD1 inhibitor might be a novel epigenetic strategy for treatment of DLBCL patients.

We developed a novel and potent LSD1 inhibitor ZY0511 [9], and found it inhibited the proliferation of various cancer cells, including hematological malignancies, cervical cancer cells, melanoma cells, and potentiated the sensitivity of 5-FU to colorectal cancer cells [18]. Here, we confirmed that ZY0511 inhibited DLBCL cells growth both in vitro and in vivo. Similar with our results, CC-90011, an oral LSD1 inhibitor, is undergoing clinical trials for NHL therapy.

Cancer is characterized by aberrant cell cycle. Cyclin-dependent kinases (CDKs) interact with cyclin to regulate cell cycle progression and proliferation [19]. Cyclin D-CDK4/CDK6 complex promotes cells progression from G0/G1 phase to S phase, at which time DNA replication begins. It has been shown that LSD1 demethylated H3K9me2 and thus inhibited expression of cell cycle-related genes, which facilitates the expression of S-phase genes and G1-S phase transition [20]. The genetic knockdown or pharmacological inhibition of LSD1 induces G0/G1 cell cycle arrest [20]. Consistent with above studies, we found that ZY0511 blocked cell cycle at G0/G1 phase. Thus, ZY0511 may regulated H3K9me2 by inhibiting LSD1, and directly inhibited CDK4 and Cyclin D1 led to arrest of G0/G1 cell cycle.

Moreover, previous studies have identified that p53 is the non-histone substrate of LSD1, which prevents p53 binding to DNA by maintaining p53 in an inactive state [21]. Kuang's group confirmed that p53-p21 pathway activation leads to CDK4 inhibition, thus resulting G1 cell cycle arrest [22]. The current study showed that ZY0511 treatment resulted G1/S transition abnormity and increased the levels of p53 and pp53. Thus, ZY0511 may inhibit Cyclin D1 expression and CDK4 expression though the activation of p53, thus disrupt the relevant cyclin D-CDK4 complex. The cell cycle progression was blocked, further leading to inhibition of cell proliferation in vitro.

It is well known that cells with cell cycle arrest eventually progress to apoptosis. Apoptosis plays a crucial role in maintaining cellular homeostasis and controlling the cell proliferation [23]. There are two major 
apoptotic pathways, the mitochondria-mediated endogenous and exogenous apoptosis, that center on the activation of caspases [24]. During the early apoptosis, the mitochondrial membrane potential which indicates the mitochondrial membrane permeability is decreased [25]. After receiving apoptosis signal, mitochondrial protein Bax relocates to the surface of the mitochondria, resulting in membrane potential decrease and permeability increase [26]. Caspase-8 is the crucial protein in the caspase family, activated in the extrinsic apoptotic pathway, resulting in activation of caspase 3 which can cause an apoptotic cascade [27]. Once LSD1 interacts with p53, both the transcriptional activation and pro-apoptosis effects of p53 will be inhibited $[21,28]$. In this study, JC- 1 analysis implied that ZY0511 activated mitochondrialmediated intrinsic apoptosis which supported by mitochondrial depolarization and pro-apoptotic protein Bax upregulation. Meanwhile, cleaved caspase 8 and cleaved caspase 3 was increased after ZY0511 treatment, suggesting that inhibition of LSD1 leads to both intrinsic and extrinsic apoptosis. These results are consistent with previous findings. Pharmacological targeting of LSD1 by pargyline or tranylcypromine induced apoptosis in oral squamous cell carcinoma cells [29]. The silencing of LSD1 also resulted apoptosis in JeKo-1 and MOLT-4 cells [30]. Furthermore, Z-VAD-FMK, a pan-caspase inhibitor, obviously rescued ZY0511-induced apoptosis.

Together with apoptosis, autophagy involves in controlling cell fate. Autophagy is originally identified as a cell survival mechanism, sequestering, degrading and recycling cellular material. Accumulating studies suggest that autophagy is a double-sided sword. Autophagy not only serve protective function via removing cellular trash, but also facilitate cell death by providing a scaffold for the cell death machinery [31]. Xu's study reported that histone lysine methyltransferase 2 inhibitor, BIX-01294, inhibits DLBCL cells proliferation by inducing endoplasmic reticulum stress mediated autophagy [32]. Another study showed that inhibiting IncRNA MALAT-1 decreases the chemotherapy resistance of DLBCL by activating autophagy [33]. Therefore, it is feasible to mediate the death of DLBCL cells by activating autophagy. Increasing studies identified that inhibition of LSD1 by knockdown or pharmacological inhibition, mediates autophagy and contributes to cell death [34-36]. LSD1 inhibits autophagy in neuroblastoma by SESN2 (Sestrin2)-dependent pathway [37]. Inhibition of LSD1 activates autophagy by stabilizing p62, thus reduces malignant cell growth [38]. Our data clearly showed that ZY0511 increased LC3-II/LC3-I ratio, the p62 expression, and autophagosomes formation in DLBCL cells, suggesting that ZY0511 significantly induced autophagy. This data is also consistent with our previous study that ZY0511 may induces cell death partly by inducing autophagy in Hela and HCT116 cells [39]. Similarly, cells dying by autophagy contains degraded intracellular membranes and cytoplasmic material and mitochondria [40], which is also involved in our results. As shown in Fig. 6d, there are enlarged mitochondria in the ZY0511 treatment group. ZY0511 may induce excessive autophagy and disturb the autophagy-lysosome flux resulting endoplasmic reticulum (ER) stress, which finally induced cell death in the tumor tissue.

In cancer cells, PI3K/AKT/mTOR pathway is the most common activated signal pathways, which promotes TORC1 activation and results autophagy inhibition [41, 42]. Our results indicated that ZY0511 obviously suppresses mTOR/P70S6K axis by inhibiting the level of phosphorylation of mTOR, p70S6K and S6. This data is consistent with our previous findings that ZY0511 predominately elevates the level 
of DDIT4, a suppressor of mTORC1 pathway and inhibits mTOR/P70S6K axis, resulting in cancer cell growth inhibition.

Several studies have suggested that inhibition of autophagy could reduce anti-tumor $T$ cell responses [43], because the anti-tumor immune system recognition and response need autophagy of dying tumor cells. Shi's group reported that inactivation of LSD1 can recruit T cells to accumulate to the tumor sites [44]. We speculate that ZY0511 may activate the recruitment of T cells into the tumor site by inhibiting LSD1 and inducing autophagy. However, substantial research is needed to further explore whether ZY0511 can stimulate anti-tumor immune response by pharmacologically inhibiting LSD1.

Combinational strategy is very attractive for DLBCL treatment. Besides overexpression of LSD1, DLBCL is characterized by downregulation of the acetyltransferases EP300/CREBBP, histone methyltransferase MLL2 and histone methyltransferase EZH2, BCL-6 dysregulation, NF-KB activation, and Ga13-dependent pathway inactivation [3]. Apart from standard treatment strategy, some anti-tumor targeted drugs currently undergo clinical trials for therapy of DLBCL, including BTK inhibitor (Ibrutinib), BCL2 inhibitor (Venetoclax), JAK1/JAK2 inhibitor (Ruxolitinib), immunomodulatory drug (Lenalidomide), anti-PD-1 antibody (Pidilizumab), and epigenetic-modifying drugs (BRD inhibitor OTX015 and EZH2 inhibitor Tazemetostat) [45]. Continued study is needed to investigate the combinatorial strategy of ZY0511 with the above inhibitors would be effective for DLBCL treatment.

In summary, we have demonstrated that ZY0511 shows anti-tumor effect against human DLBCL cells by blocking G0/G1 cell cycle progress and triggering apoptosis. Additionally, ZY0511 induced autophagy though mTOR/P70S6K axis. Moreover, ZY0511 exhibited tumor growth in vivo without adverse events. Therefore, our findings suggest that ZY0511 might be a novel and promising treatment strategy for treating DLBCL.

\section{Declarations}

\section{Funding}

This study was supported by the National Natural Sciences Foundation of China (81773198) and the National S\&T Major Project (2018ZX09201018).

\section{Competing Interests}

The authors have declared that no competing interest exists.

\section{Authors' contributions}

Writing-Review \& Editing: HL, NS and YZ: Original Draft; Supervision and Writing: YZ, YY and JD; Methodology and Data Curation: HL, JW, XZ, XZ XY and JZ; Research design: ZZ and YZ. 


\section{Ethics statement}

The animal study was reviewed and approved by the Ethics Committee of Sichuan University.

\section{References}

1. Miao Y, Medeiros LJ, Li Y, Li J, Young KH. Genetic alterations and their clinical implications in DLBCL. Nat Rev Clin Oncol. 2019; 16(10): 634-52.

2. Pfreundschuh $M$, Trümper $L$, Osterborg $A$, Pettengell $R$, Trneny $M$, Imrie $K$, et al. CHOP-like chemotherapy plus rituximab versus $\mathrm{CHOP}$-like chemotherapy alone in young patients with goodprognosis diffuse large-B-cell lymphoma: a randomised controlled trial by the MabThera International Trial (MInT) Group. Lancet Oncol. 2006; 7(5): 379-91.

3. Basso K, Dalla-Favera R. Germinal centres and B cell lymphomagenesis. Nat Rev Immunol. 2015; 15(3): 172-84.

4. Shi Y, Lan F, Matson C, Mulligan P, Whetstine JR, Cole PA, et al. Histone Demethylation Mediated by the Nuclear Amine Oxidase Homolog LSD1. Cell. 2004; 119(7): 941-53.

5. Metzger E, Wissmann M, Yin N, Müller JM, Schneider R, Peters AH, et al. LSD1 demethylates repressive histone marks to promote androgen-receptor-dependent transcription. Nature. 2005; 437(7057): 436-9.

6. Carmichael CL, Wang J, Nguyen T, Kolawole O, Benyoucef A, De Mazière $C$, et al. The EMT modulator SNAl1 contributes to AML pathogenesis via its interaction with LSD1. Blood. 2020; 136(8): 957-73.

7. Hatzi K, Geng H, Doane AS, Meydan C, LaRiviere R, Cardenas M, et al. Histone demethylase LSD1 is required for germinal center formation and BCL6-driven lymphomagenesis. Nat Immunol. 2019; 20(1): 86-96.

8. Fang Y, Liao G, Yu B. LSD1/KDM1A inhibitors in clinical trials: advances and prospects. J Hematol Oncol. 2019; 12(1): 129.

9. Zhou Y, Li Y, Wang WJ, Xiang P, Luo XM, Yang L, et al. Synthesis and biological evaluation of novel (E)-N'-(2,3-dihydro-1H-inden-1-ylidene) benzohydrazides as potent LSD1 inhibitors. Bioorg Med Chem Lett. 2016; 26(18): 4552-7.

10. Xia Y, Xu F, Xiong M, Yang H, Lin W, Xie Y, et al. Repurposing of antipsychotic trifluoperazine for treating brain metastasis, lung metastasis and bone metastasis of melanoma by disrupting autophagy flux. Pharmacol Res. 2021; 163: 105295.

11. Martinez Molina D, Jafari R, Ignatushchenko M, Seki T, Larsson EA, Dan C, et al. Monitoring drug target engagement in cells and tissues using the cellular thermal shift assay. Science. 2013; 341(6141): 84-7.

12. Wei MC, Zong WX, Cheng EH, Lindsten T, Panoutsakopoulou V, Ross AJ, et al. Proapoptotic BAX and BAK: a requisite gateway to mitochondrial dysfunction and death. Science. 2001; 292(5517): 727-30. 
13. Zorova LD, Popkov VA, Plotnikov EY, Silachev DN, Pevzner IB, Jankauskas SS, et al. Mitochondrial membrane potential. Anal Biochem. 2018; 552(50-9.

14. He M, Zhang T, Zhu Z, Qin S, Wang H, Zhao L, et al. LSD1 contributes to programmed oocyte death by regulating the transcription of autophagy adaptor SQSTM1/p62. Aging Cell. 2020; 19(3).

15. Xu Z, Han X, Ou D, Liu T, Li Z, Jiang G, et al. Targeting PI3K/AKT/mTOR-mediated autophagy for tumor therapy. Appl Microbiol Biotechnol. 2019; 104(2): 575-87.

16. Meyer SN, Scuoppo C, Vlasevska S, Bal E, Holmes AB, Holloman M, et al. Unique and Shared Epigenetic Programs of the CREBBP and EP300 Acetyltransferases in Germinal Center B Cells Reveal Targetable Dependencies in Lymphoma. Immunity. 2019; 51(3): 535-47 e9.

17. Brach D, Johnston-Blackwell D, Drew A, Lingaraj T, Motwani V, Warholic NM, et al. EZH2 Inhibition by Tazemetostat Results in Altered Dependency on B-cell Activation Signaling in DLBCL. Mol Cancer Ther. 2017; 16(11): 2586-97.

18. Peng W, Zhang H, Tan S, Li Y, Zhou Y, Wang L, et al. Synergistic antitumor effect of 5-fluorouracil with the novel LSD1 inhibitor ZY0511 in colorectal cancer. Ther Adv Med Oncol. 2020; 12 : 1758835920937428.

19. Sherr CJ, Roberts JM. Living with or without cyclins and cyclin-dependent kinases. Genes Dev. 2004; 18(22): 2699-711.

20. He Y, Zhao Y, Wang L, Bohrer LR, Pan Y, Wang L, et al. LSD1 promotes S-phase entry and tumorigenesis via chromatin co-occupation with E2F1 and selective H3K9 demethylation. Oncogene. 2018; 37(4): 534-43.

21. Huang J, Sengupta R, Espejo AB, Lee MG, Dorsey JA, Richter M, et al. p53 is regulated by the lysine demethylase LSD1. Nature. 2007; 449(7158): 105-8.

22. He G, Siddik ZH, Huang Z, Wang R, Koomen J, Kobayashi R, et al. Induction of p21 by p53 following DNA damage inhibits both Cdk4 and Cdk2 activities. Oncogene. 2005; 24(18): 2929-43.

23. Li K, Deng Y, Deng G, Chen P, Wang Y, Wu H, et al. High cholesterol induces apoptosis and autophagy through the ROS-activated AKT/FOXO1 pathway in tendon-derived stem cells. Stem Cell Res Ther. 2020; 11(1): 131.

24. Shalini S, Dorstyn L, Dawar S, Kumar S. Old, new and emerging functions of caspases. Cell Death Differ. 2015; 22(4): 526-39.

25. Zhu YY, Huang HY, Wu YL. Anticancer and apoptotic activities of oleanolic acid are mediated through cell cycle arrest and disruption of mitochondrial membrane potential in HepG2 human hepatocellular carcinoma cells. Mol Med Rep. 2015; 12(4): 5012-8.

26. Shimizu S, Narita M, Tsujimoto Y. Bcl-2 family proteins regulate the release of apoptogenic cytochrome $\mathrm{c}$ by the mitochondrial channel VDAC. Nature. 1999; 399(6735): 483-7.

27. Taylor RC, Cullen SP, Martin SJ. Apoptosis: controlled demolition at the cellular level. Nat Rev Mol Cell Biol. 2008; 9(3): 231-41. 
28. Vadnais C, Chen R, Fraszczak J, Hamard PJ, Manfredi JJ, Moroy T. A novel regulatory circuit between p53 and GFI1 controls induction of apoptosis in T cells. Sci Rep. 2019; 9(1): 6304.

29. Wang Y, Zhu Y, Wang Q, Hu H, Li Z, Wang D, et al. The histone demethylase LSD1 is a novel oncogene and therapeutic target in oral cancer. Cancer Lett. 2016; 374(1): 12-21.

30. Zou ZK, Huang YQ, Zou Y, Zheng XK, Ma XD. Silencing of LSD1 gene modulates histone methylation and acetylation and induces the apoptosis of JeKo-1 and MOLT-4 cells. Int J Mol Med. 2017; 40(2): 319-28.

31. Doherty J, Baehrecke EH. Life, death and autophagy. Nat Cell Biol. 2018; 20(10): 1110-7.

32. Xu L, Gao X, Yang P, Sang W, Jiao J, Niu M, et al. EHMT2 inhibitor BIX-01294 induces endoplasmic reticulum stress mediated apoptosis and autophagy in diffuse large B-cell lymphoma cells. J Cancer. 2021; 12(4): 1011-22.

33. Li LJ, Chai Y, Guo XJ, Chu SL, Zhang LS. The effects of the long non-coding RNA MALAT-1 regulated autophagy-related signaling pathway on chemotherapy resistance in diffuse large B-cell lymphoma. Biomed Pharmacother. 2017; 89: 939-48.

34. Feng S, Jin Y, Cui M, Zheng J. Lysine-Specific Demethylase 1 (LSD1) Inhibitor S2101 Induces Autophagy via the AKT/mTOR Pathway in SKOV3 Ovarian Cancer Cells. Med Sci Monit. 2016; 22: 4742-8.

35. Liu S, Lu W, Li S, Li S, Liu J, Xing Y, et al. Identification of JL1037 as a novel, specific, reversible lysine-specific demethylase 1 inhibitor that induce apoptosis and autophagy of AML cells. Oncotarget. 2017; 8(19): 31901-14.

36. Wang Z, Long QY, Chen L, Fan JD, Wang ZN, Li LY, et al. Inhibition of H3K4 demethylation induces autophagy in cancer cell lines. Biochim Biophys Acta Mol Cell Res. 2017; 1864(12): 2428-37.

37. Ambrosio S, Saccà CD, Amente S, Paladino S, Lania L, Majello B. Lysine-specific demethylase LSD1 regulates autophagy in neuroblastoma through SESN2-dependent pathway. Oncogene. 2017; 36(48): 6701-11.

38. Chao A, Lin CY, Chao AN, Tsai CL, Chen MY, Lee LY, et al. Lysine-specific demethylase 1 (LSD1) destabilizes p62 and inhibits autophagy in gynecologic malignancies. Oncotarget. 2017; 8(43): 74434-50.

39. Li Y, Tao L, Zuo Z, Zhou Y, Qian X, Lin Y, et al. ZY0511, a novel, potent and selective LSD1 inhibitor, exhibits anticancer activity against solid tumors via the DDIT4/mTOR pathway. Cancer Lett. 2019; 454: 179-90.

40. Dasari SK, Bialik S, Levin-Zaidman S, Levin-Salomon V, Merrill AH, Jr., Futerman AH, et al. Signalomewide RNAi screen identifies GBA1 as a positive mediator of autophagic cell death. Cell Death Differ. 2017; 24(7): 1288-302.

41. Neufeld TP. TOR-dependent control of autophagy: biting the hand that feeds. Curr Opin Cell Biol. 2010; 22(2): 157-68.

42. Saxton RA, Sabatini DM. mTOR Signaling in Growth, Metabolism, and Disease. Cell. 2017; 169(2): $361-71$. 
43. Townsend KN, Hughson LR, Schlie K, Poon VI, Westerback A, Lum JJ. Autophagy inhibition in cancer therapy: metabolic considerations for antitumor immunity. Immunol Rev. 2012; 249(1): 176-94.

44. Sheng W, LaFleur MW, Nguyen TH, Chen S, Chakravarthy A, Conway JR, et al. LSD1 Ablation Stimulates Anti-tumor Immunity and Enables Checkpoint Blockade. Cell. 2018; 174(3): 549-63 e19.

45. Younes A, Ansell S, Fowler N, Wilson W, de Vos S, Seymour J, et al. The landscape of new drugs in lymphoma. Nat Rev Clin Oncol. 2017; 14(6): 335-46.

\section{Figures}

a

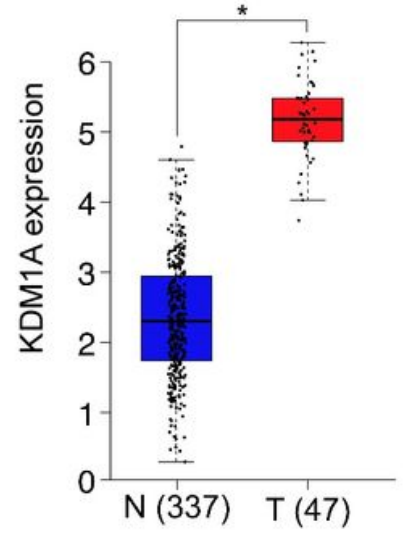

b

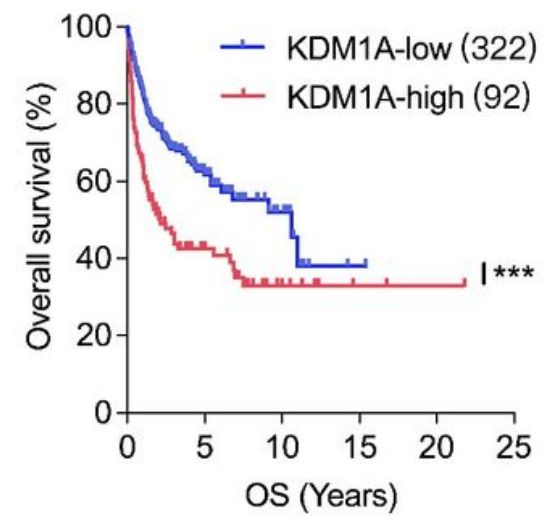

g

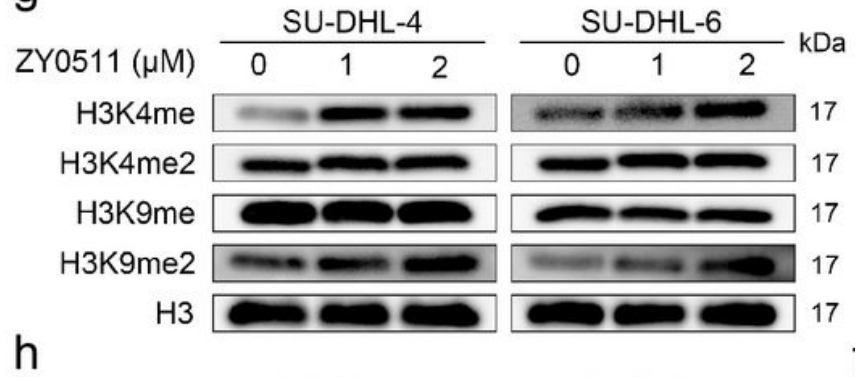

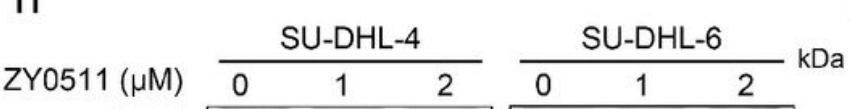

LSD1 $\square-2=110$

GAPDH 37

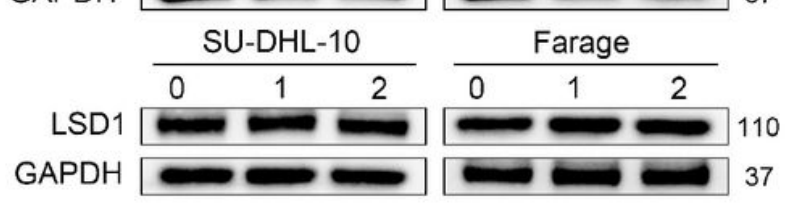

C
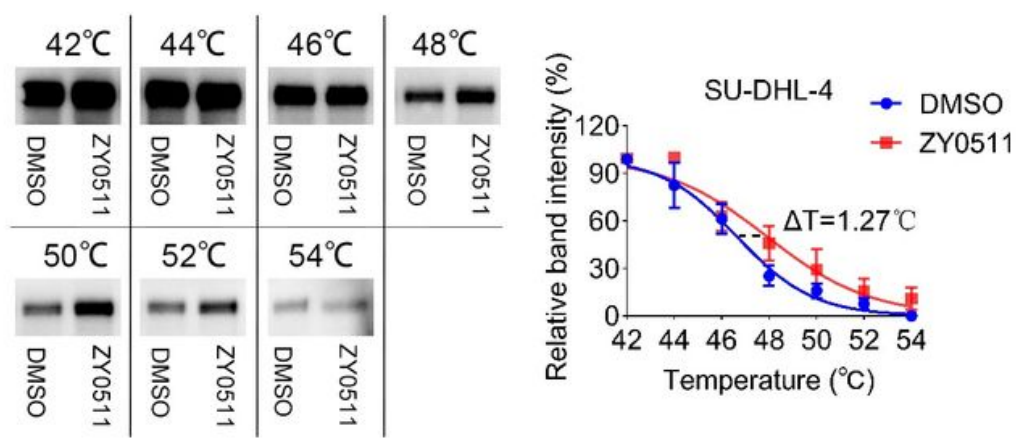

d
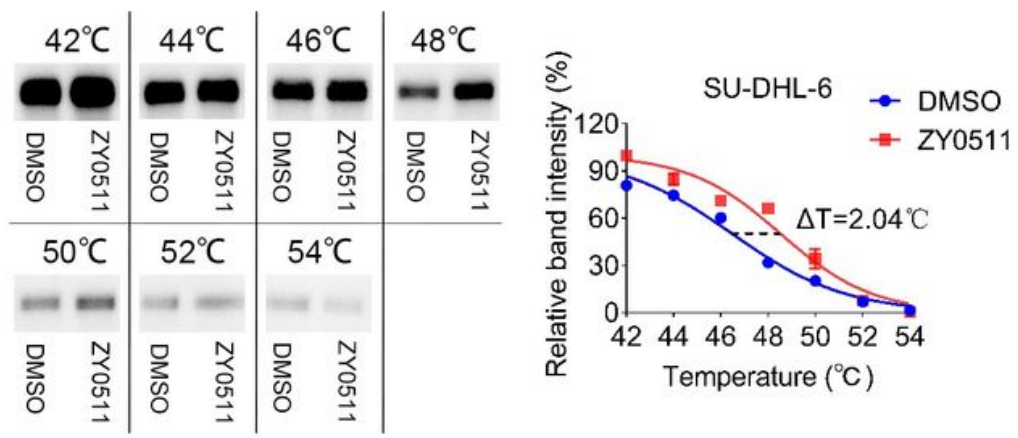

e
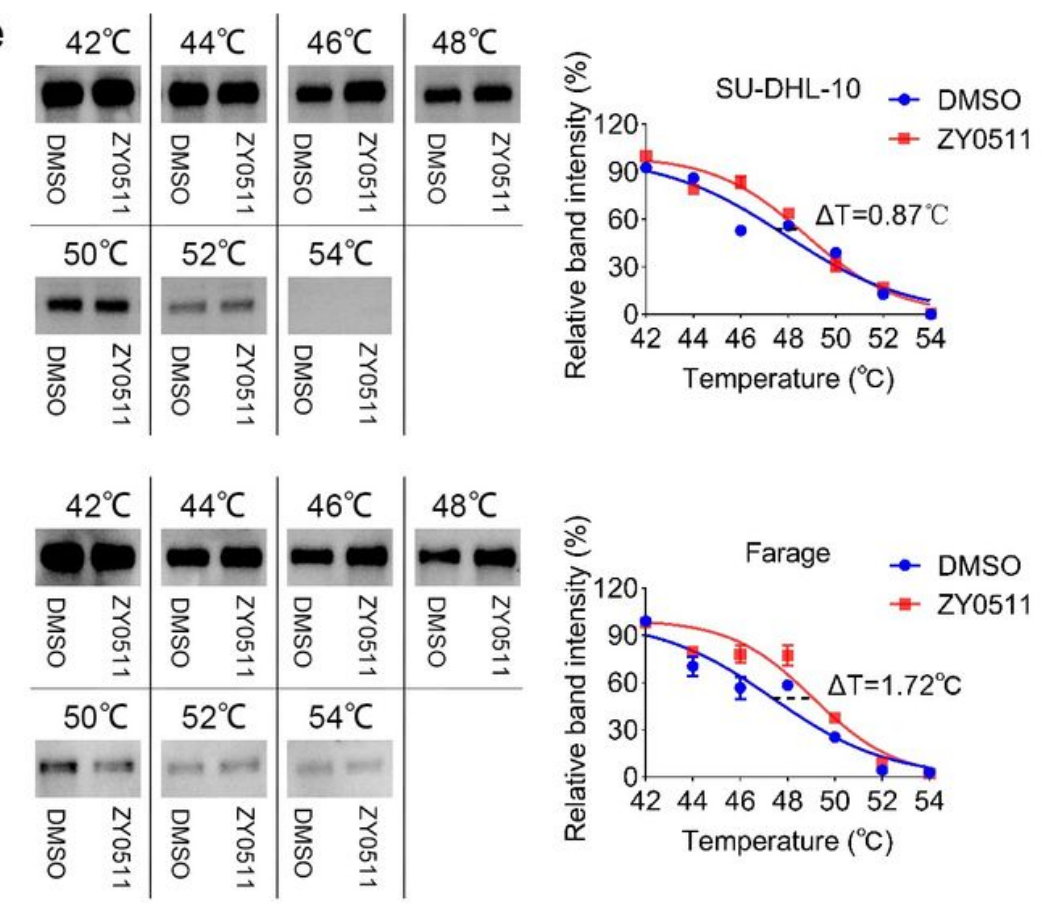


\section{Figure 1}

ZY0511 interacts with LSD1 in DLBCL Cells a The mRNA expression of KDM1A in human DLBCL tissues and normal lymph node tissues from GEPIA web ( ${ }^{*} \mathrm{P}<0.05$; student's t-test). $\mathrm{b}$ Kaplan-Meier survival analysis was used to analyze the relationship between overall survival rates of DLBCL patients and the mRNA expression of KDM1A ( $* \star * P<0.001$; log-rank test). c-f CETSA melt curve from $42^{\circ} \mathrm{C}$ to $54^{\circ} \mathrm{C}$ of DLBCL cells lysates with or without ZY0511 incubation. The representative graphs (left) and quantitative results (right) of western blot results. The data are presented as the means \pm SEM. g-h Western blot detection of H3K4meロH3K4me2ロH3K9me and H3K9me2 (G) and LSD1 (H) after ZY0511 treatment (1, 2 $\mu \mathrm{M})$. Histone $3(\mathrm{H} 3)$ is used as nucleus reference protein and GAPDH was used as the reference protein 
a

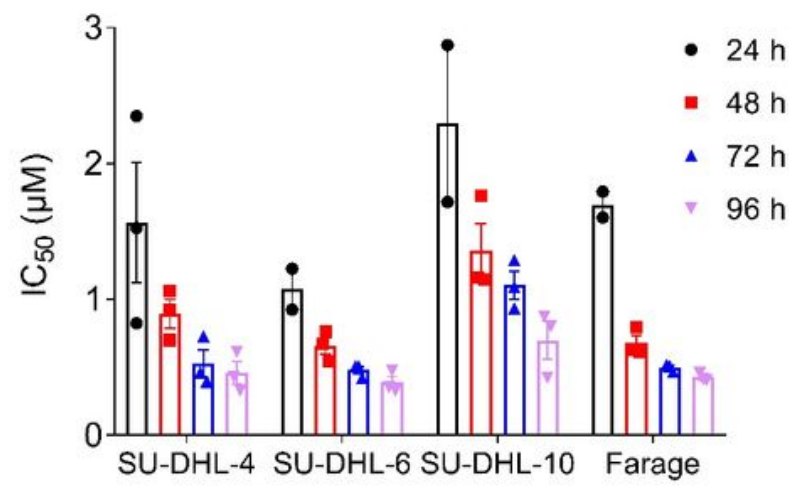

b

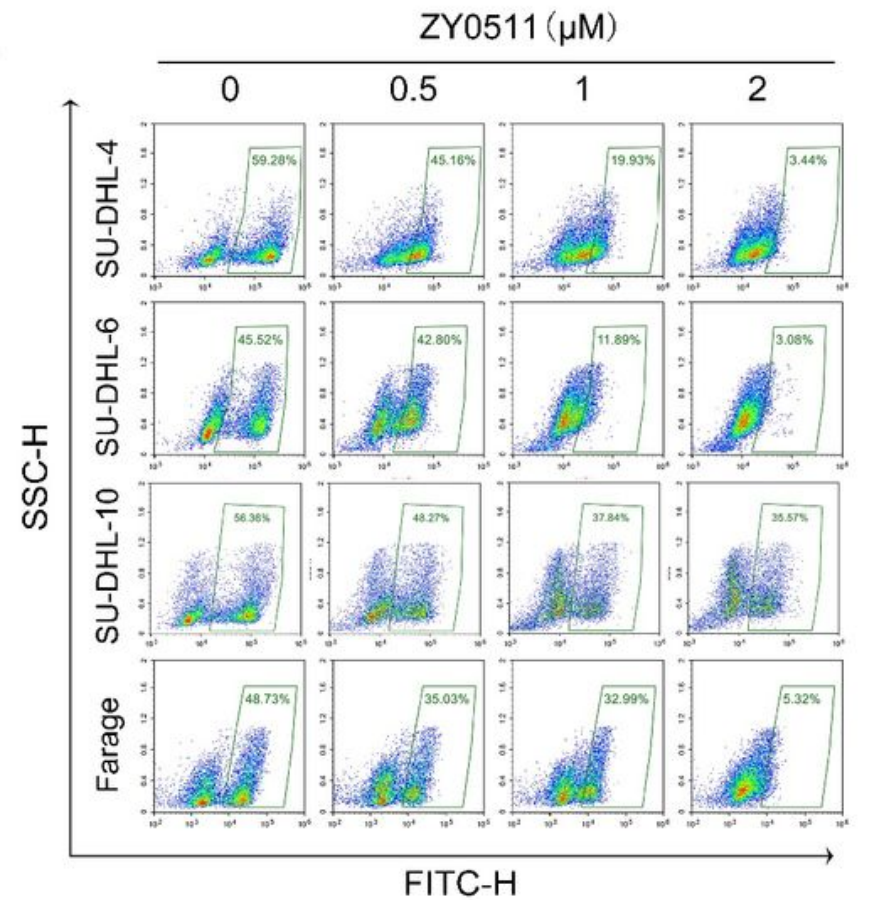

e

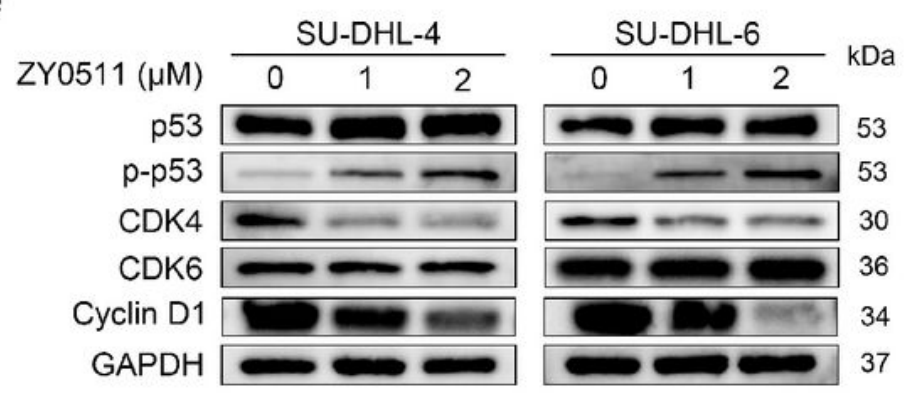

C

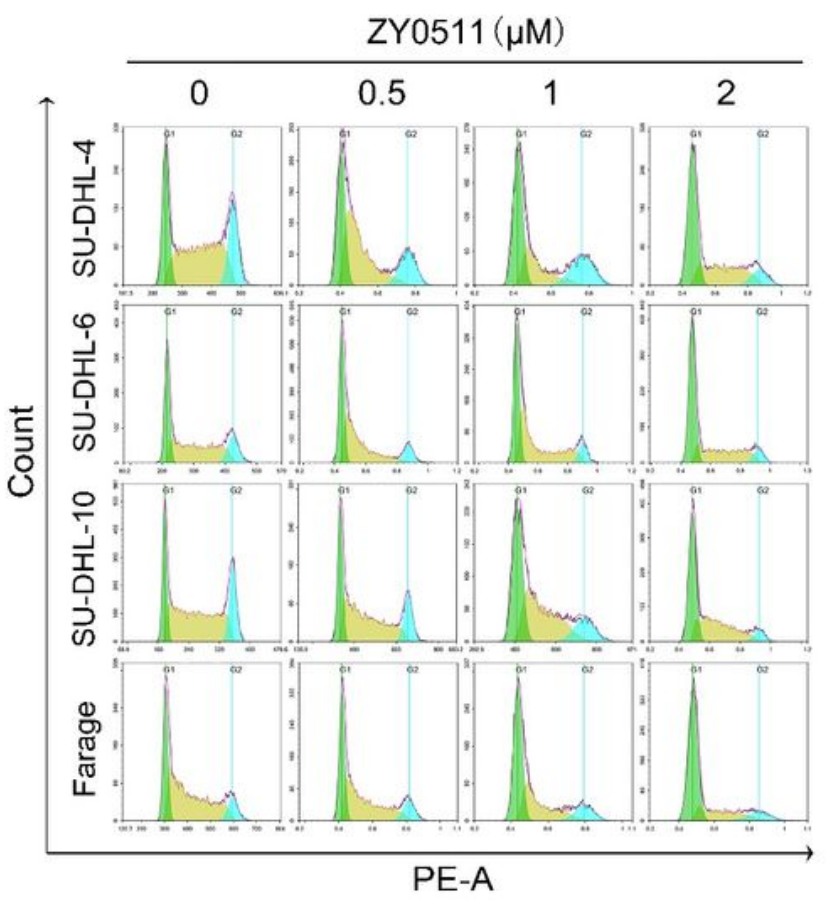

d
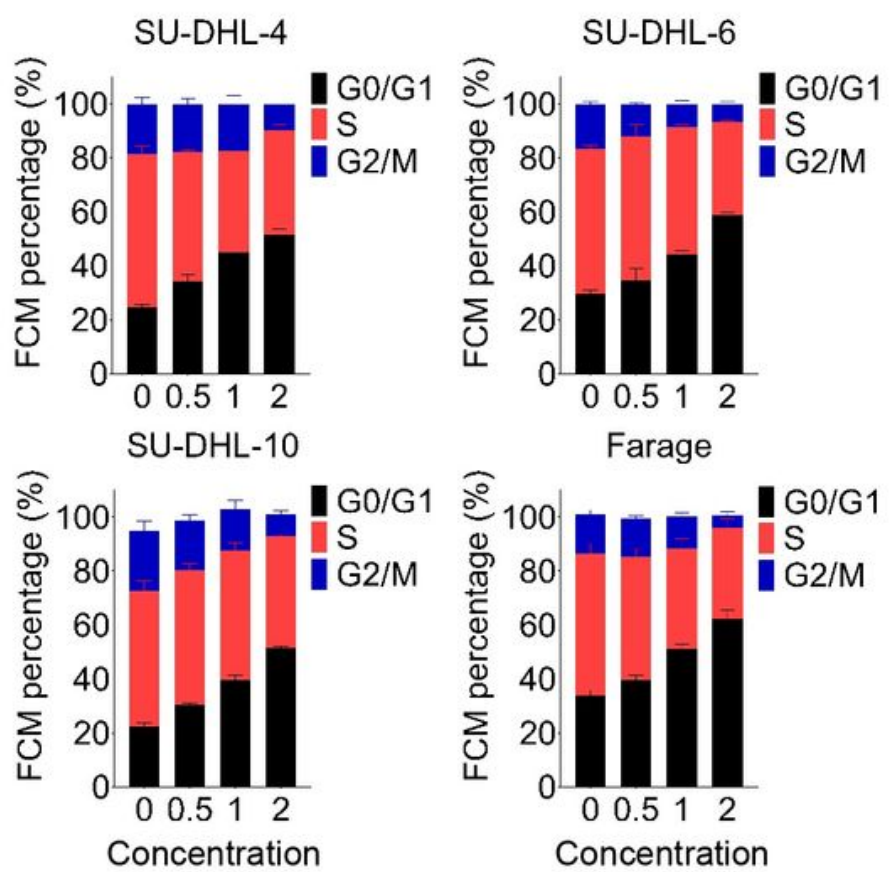

Figure 2

ZY0511 inhibits DLBCL cells proliferation in vitro a The DLBCL cells proliferation after various concentration of ZY0511 treatment for 24-96 h was detected by MTT assay. GraphPad Prism 8 was used to calculate IC50 values. b The anti-proliferation effect of ZY0511 against SU-DHL-4, SU-DHL-6, SU-DHL10 and Farage by EdU incorporation assay. c-d Cell cycle analysis of SU-DHL-4, SU-DHL-6, SU-DHL-10 and 
Farage after ZY0511 treatment. The data are presented as the means \pm SEM. e Western blot detection of cell cycle pathway proteins

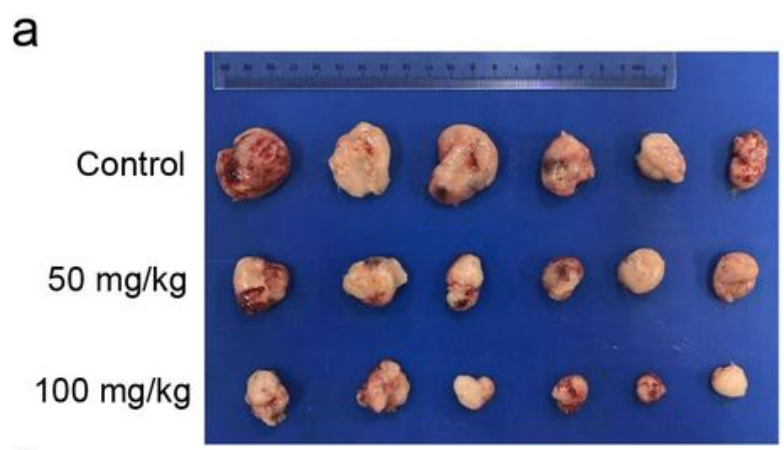

C

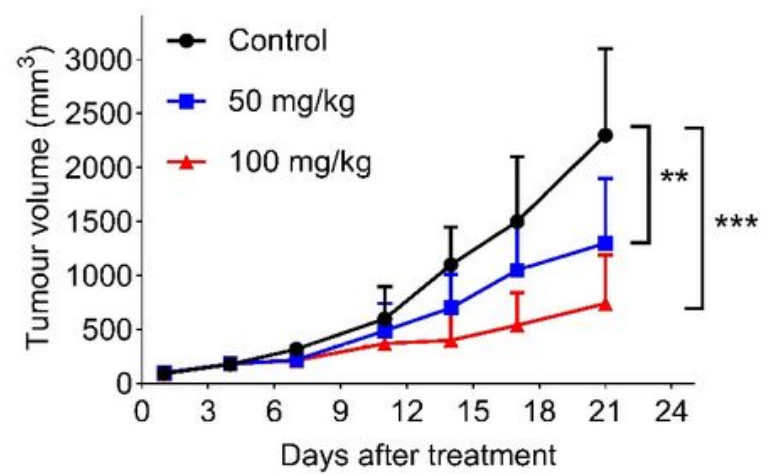

b
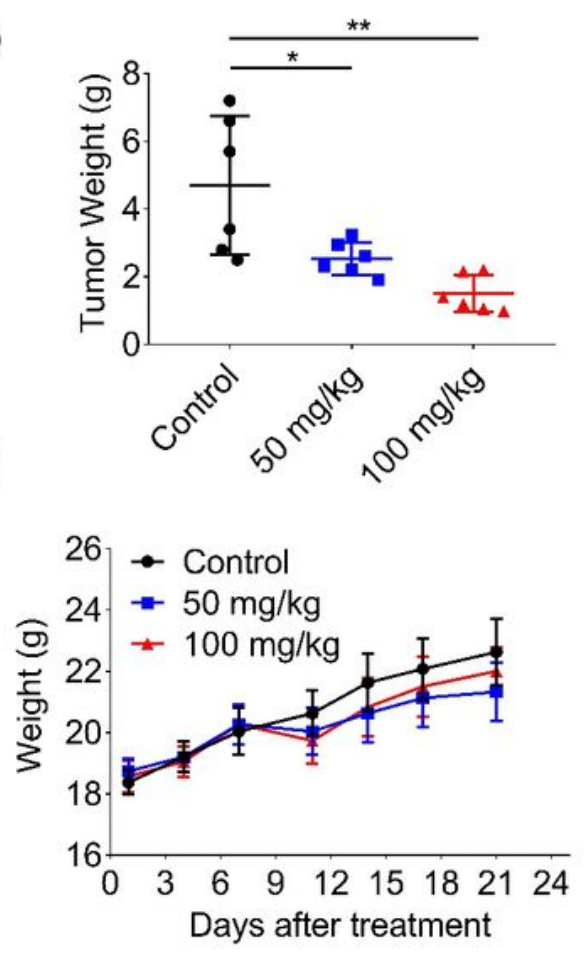

e

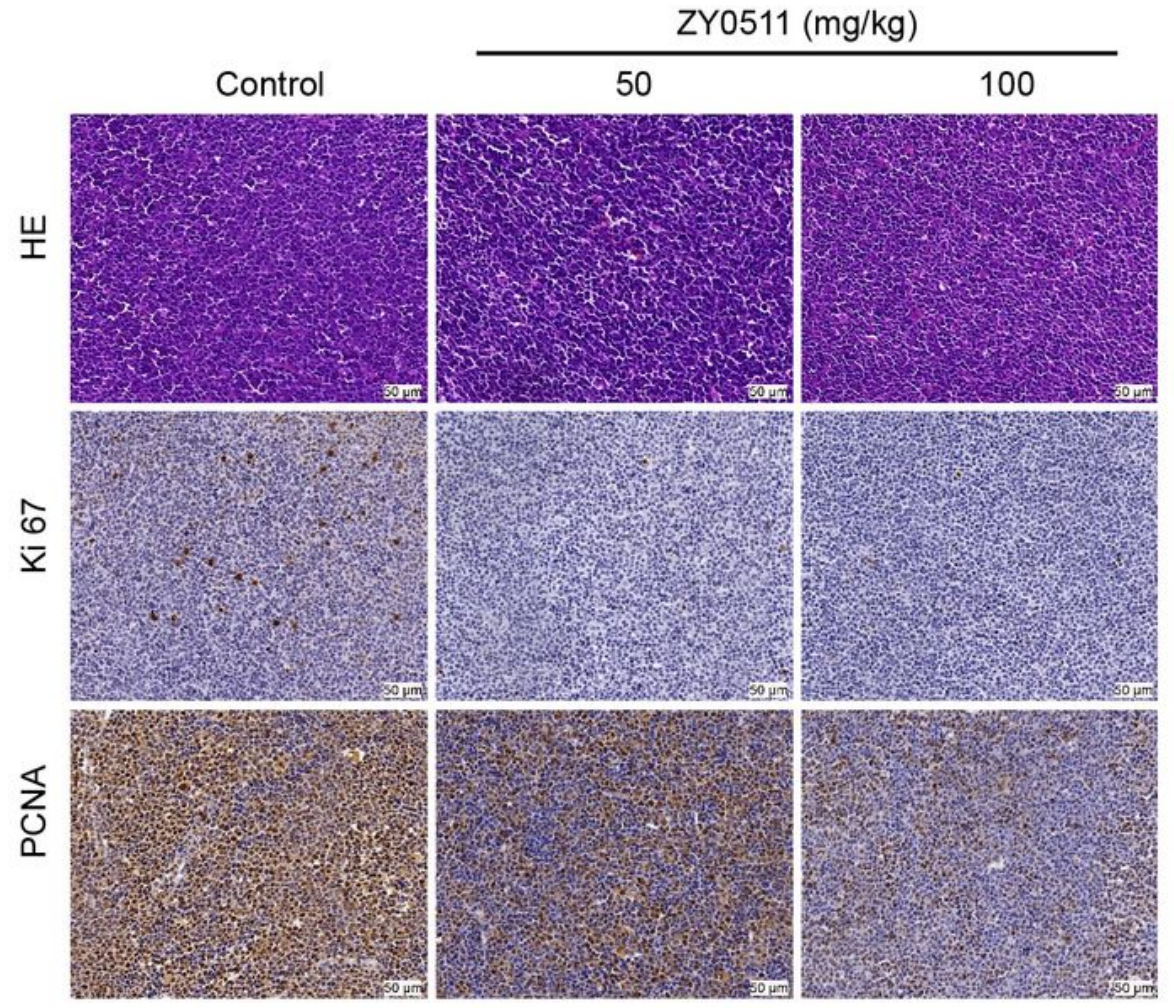

Figure 3

The antitumor effect of ZY0511 against DLBCL in vivo a-c The tumor images (A), tumor weights (B), and tumor volumes (C) for SU-DHL-6 xenograft model treated with vehicle, ZY0511 (50 mg/kg) and ZY0511 $(100 \mathrm{mg} / \mathrm{kg})$, respectively. The data are presented as the mean $\pm \mathrm{SEM}$. ${ }^{*} \mathrm{P}<0.05,{ }^{*} \mathrm{P}<0.01$, one-way 
ANOVA followed by Dunnett's test. $d$ The body weights of SU-DHL-6 xenograft model mice during treatment with ZY0511. The data are presented as the means \pm SEM. e Representative images of the H\&E, Ki67 and PCNA staining of tumors after ZY0511 treatment. Scale bar, $50 \mu \mathrm{m}$

a

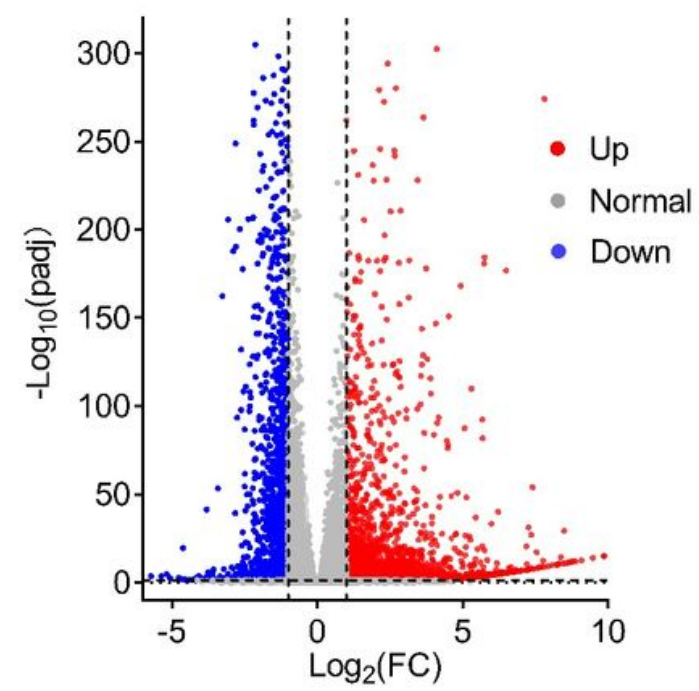

b

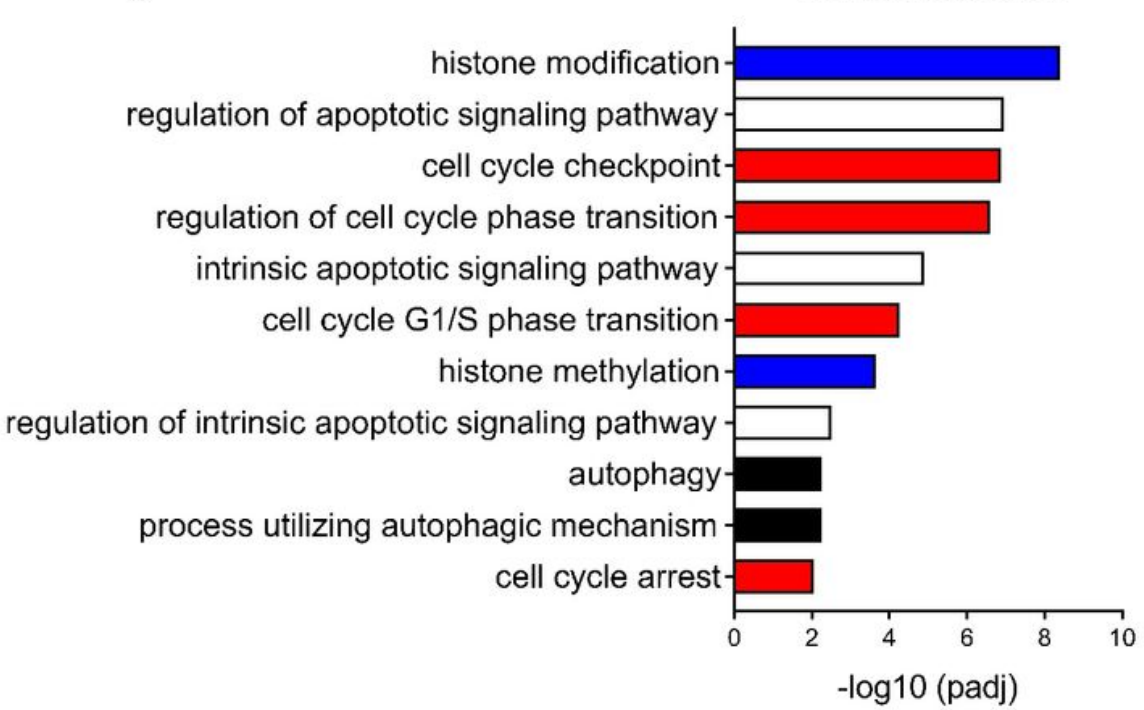

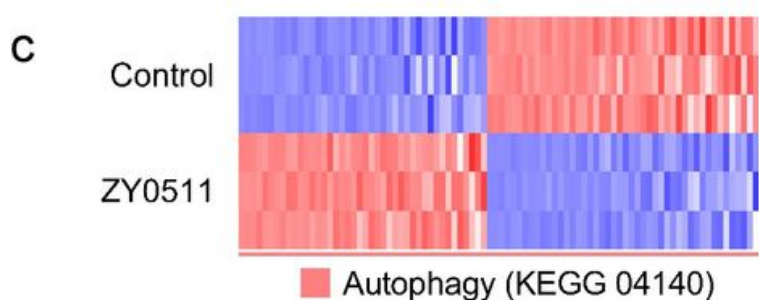

Autophagy (KEGG 04140)
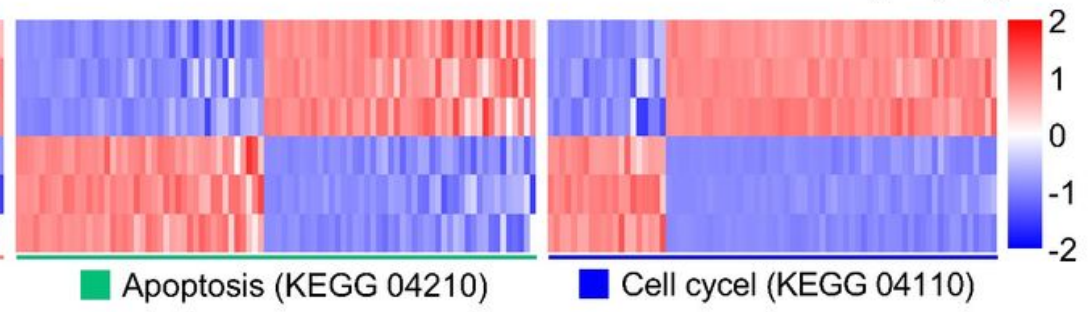

d

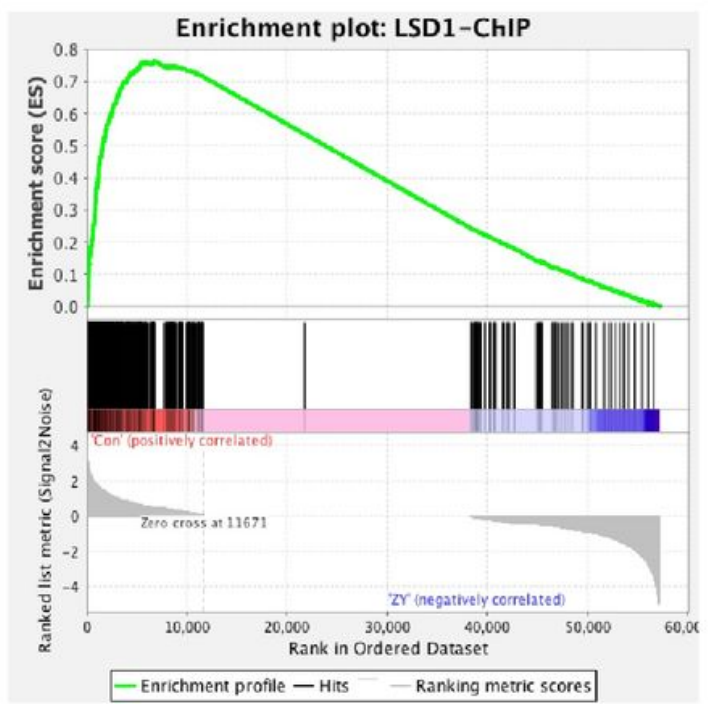

e

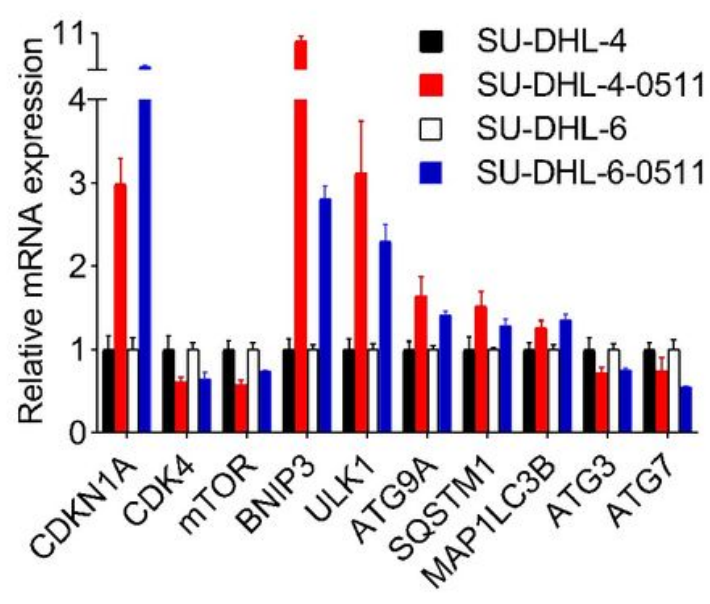

Figure 4

ZY0511 induces gene expression changes in DLBCL cells a Volcano plot of differentially expressed genes in SU-DHL-4 cells after ZY0511 treatment (from RNA-seq data). Red dots represent significantly upregulated genes, blue dots represent significantly down-regulated genes, and gray dots represent no meaningful changed genes. b Representative $\mathrm{GO}$ term analysis of significantly changed genes after ZY0511 treatment. c Heatmaps for the expression patterns of differentially expressed genes (adjusted P < 
0.05), in cell cycle, apoptosis and autophagy gene sets. d GSEA plots show enrichment of gene sets regulated following treatment of SU-DHL-4 cells with $1 \mu \mathrm{M}$ ZY0511. e qRT-PCR validation of expression changes of 10 genes selected from RNA-seq in DLBCL cells

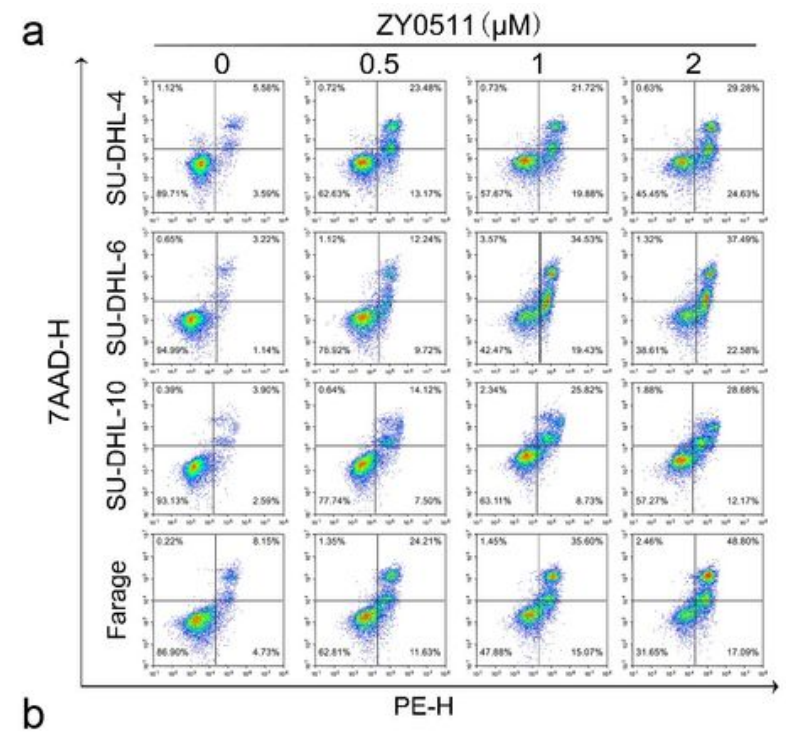

C
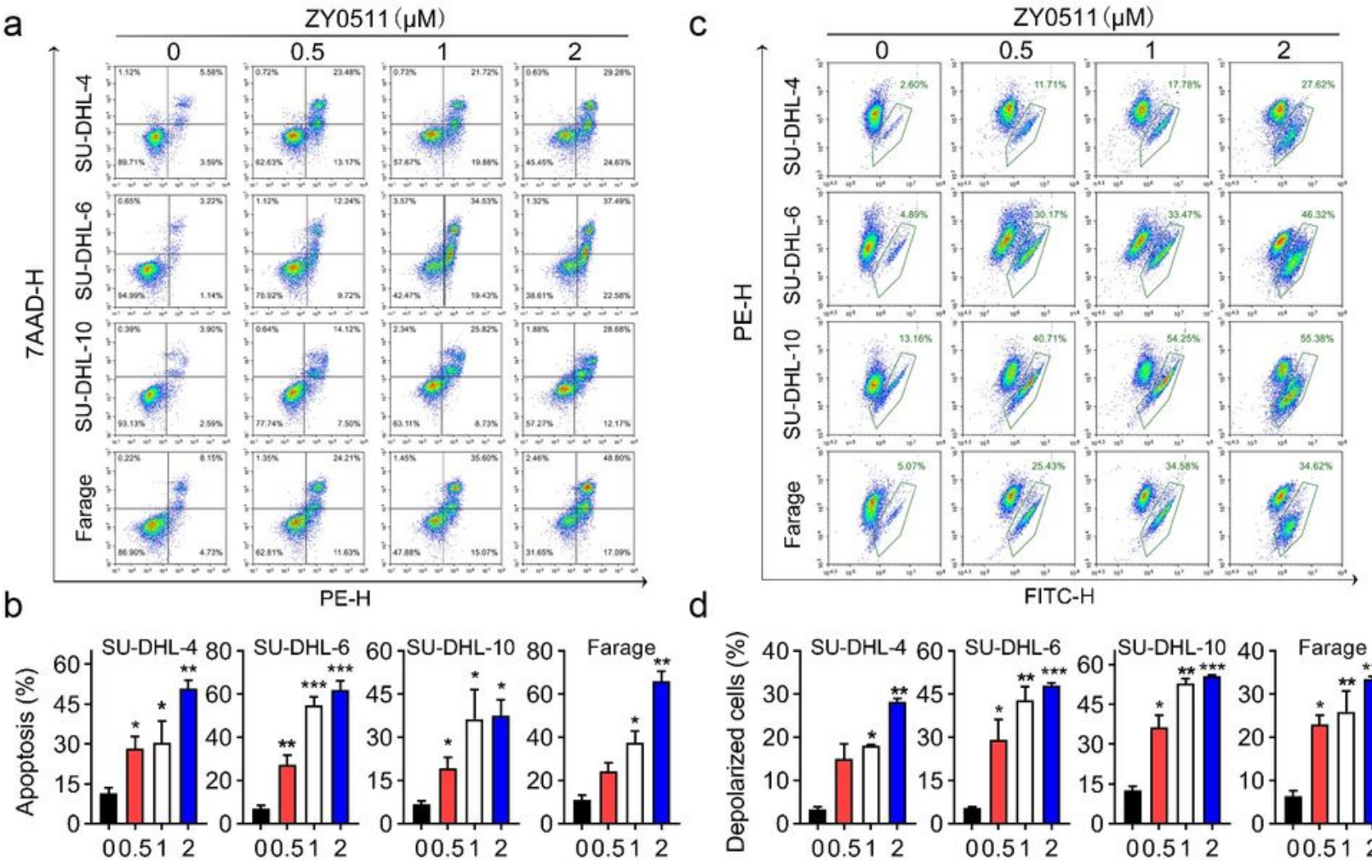

f
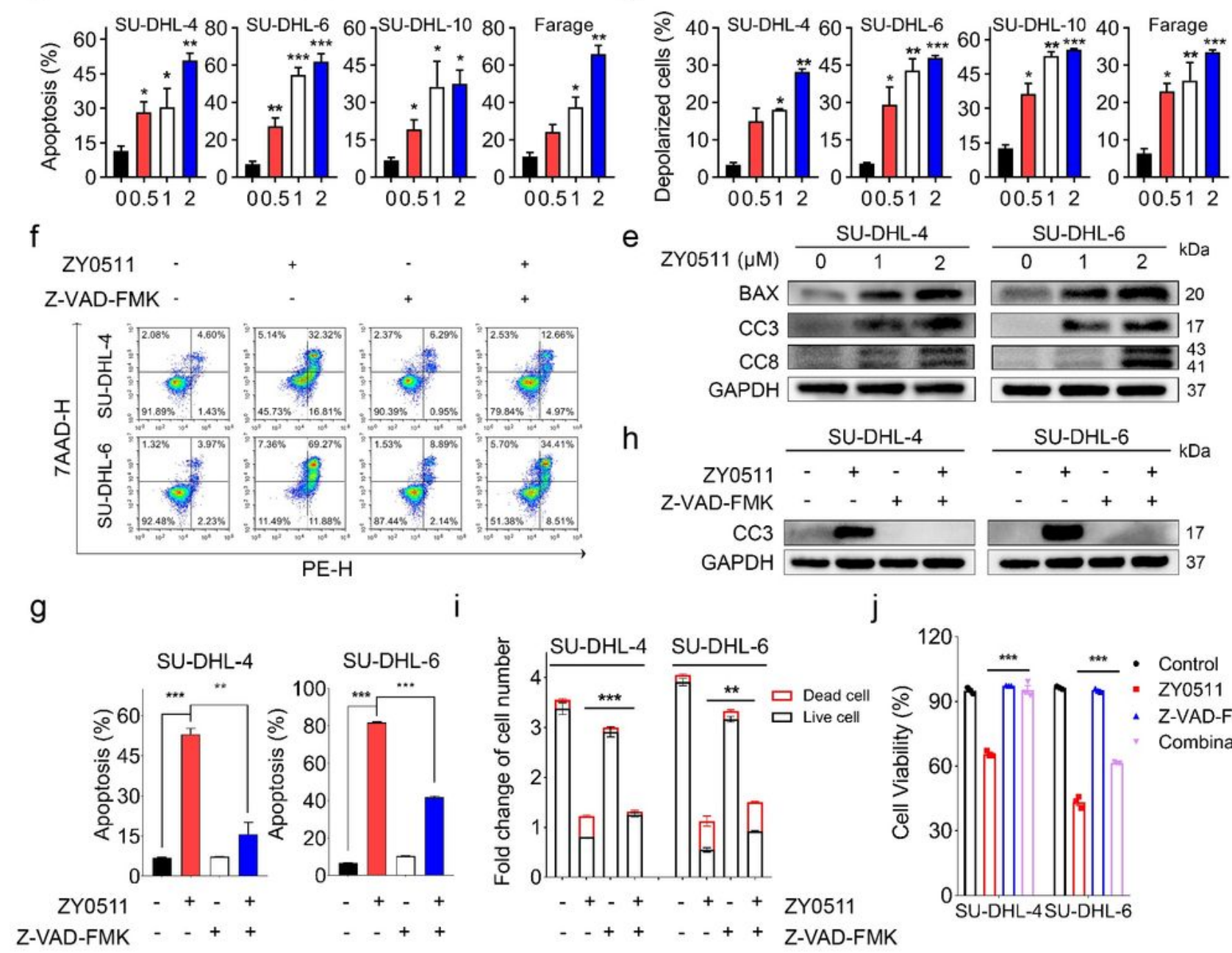

e

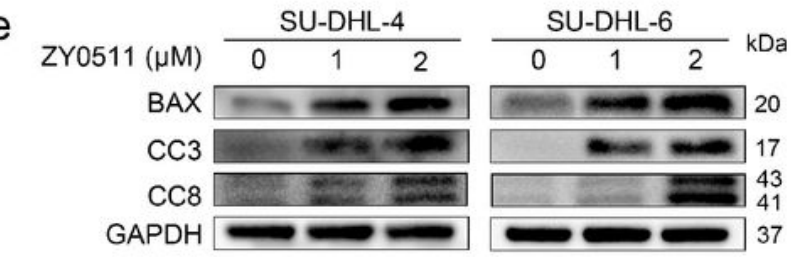

$\mathrm{h}$
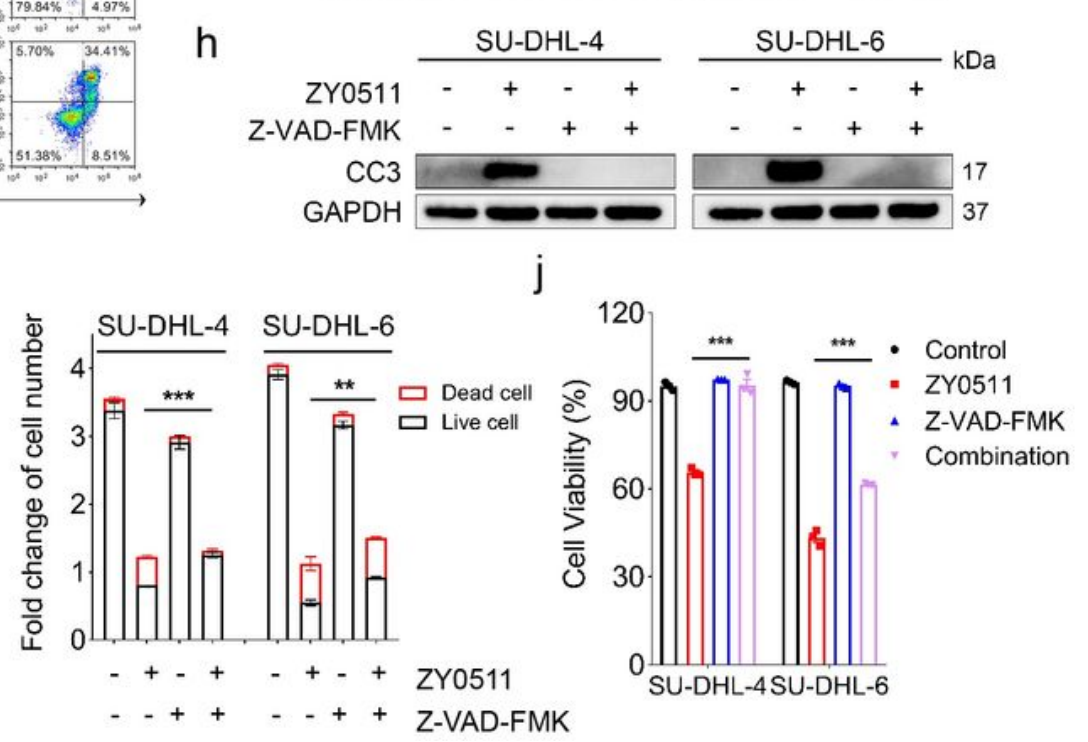

Figure 5

ZY0511 induces apoptosis in DLBCL Cells a-b Flow cytometric histograms of apoptotic DLBCL cells after ZY0511 treatment at the indicated concentrations. The data are presented as the means \pm SEM. ${ }^{*} P<0.05$, 
$\star \star P<0.01,{ }^{\star \star \star} \mathrm{P}<0.001$, one-way ANOVA followed by Dunnett's test. c-d $\Delta \Psi \mathrm{m}$ assay in DLBCL cells after ZY0511 treatment by FCM. The data are presented as the means \pm SEM. ${ }^{*}<0.05, \star \star P<0.01$, $* \star \star P<$ 0.001 , one-way ANOVA followed by Dunnett's test. e Western blot detection of BAX, CC3, and CC8 in DLBCL cells after ZY0511 treatment. f-g Flow cytometric histograms of apoptotic DLBCL cells after ZY0511 and Z-VAD-FMK cotreatment. The data are presented as the means $\pm S E M . * P<0.05, * * P<0.01$, $\star \star \star P<0.001$, one-way ANOVA followed by Dunnett's test. $\mathrm{h}$ Western blot detection of CC3 after ZY0511 and Z-VAD-FMK treatment. i-j The fold change of cell number and cell viability of DLBCL cells after the combination treatment of ZY0511 and Z-VAD-FMK by AO/PI assay. The data are presented as the means \pm SEM. ${ }^{*} P<0.05,{ }^{*} P<<0.01, * * * P<0.001$, one-way ANOVA followed by Dunnett's test

a

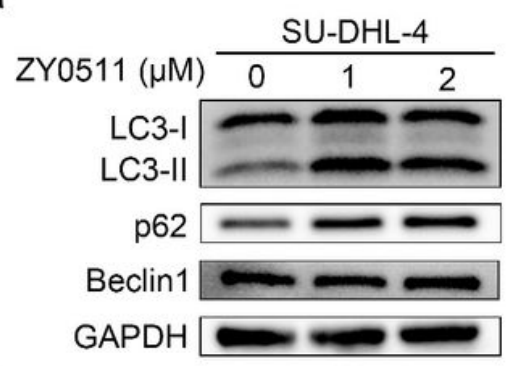

b

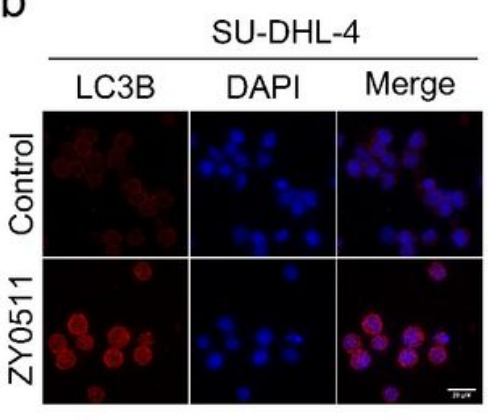

C
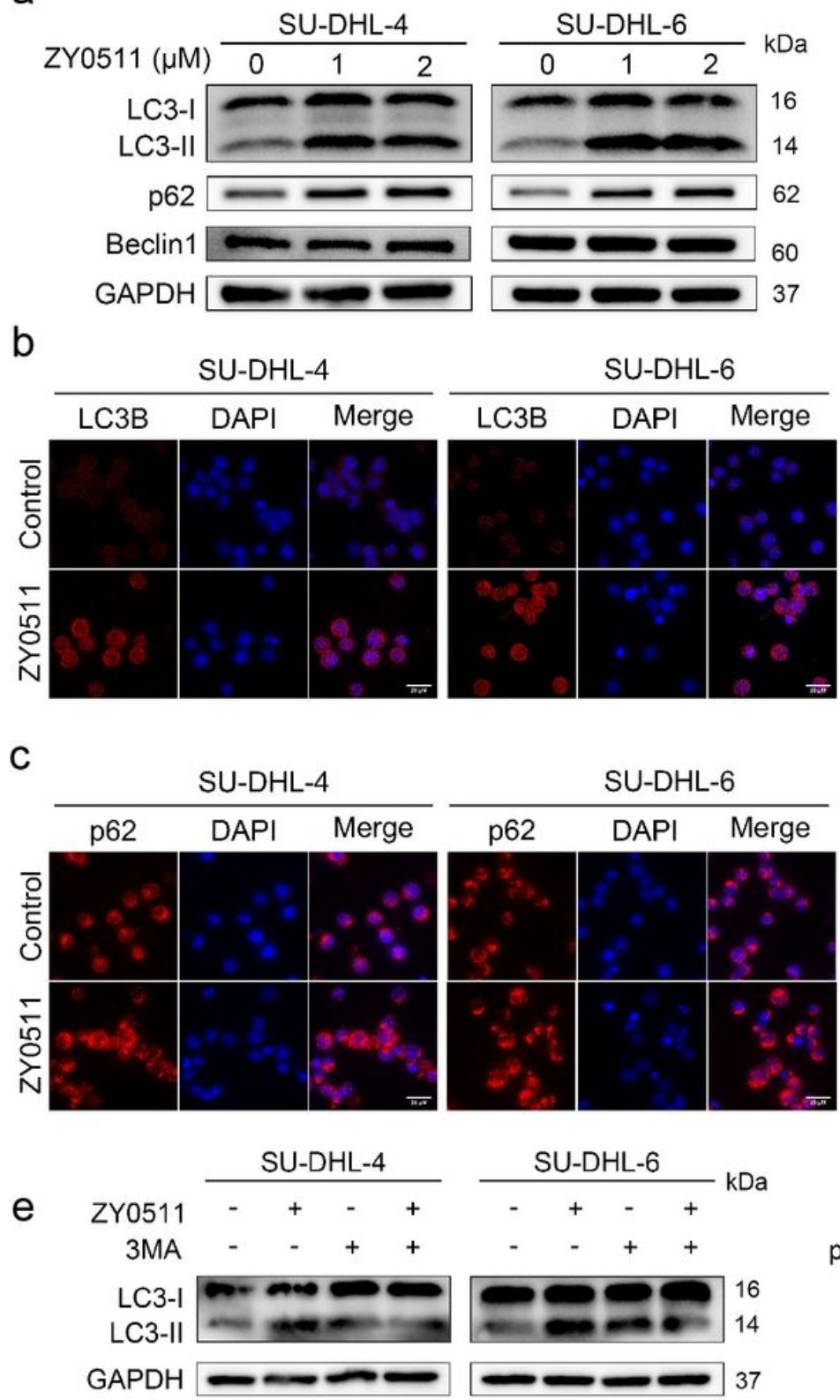

d
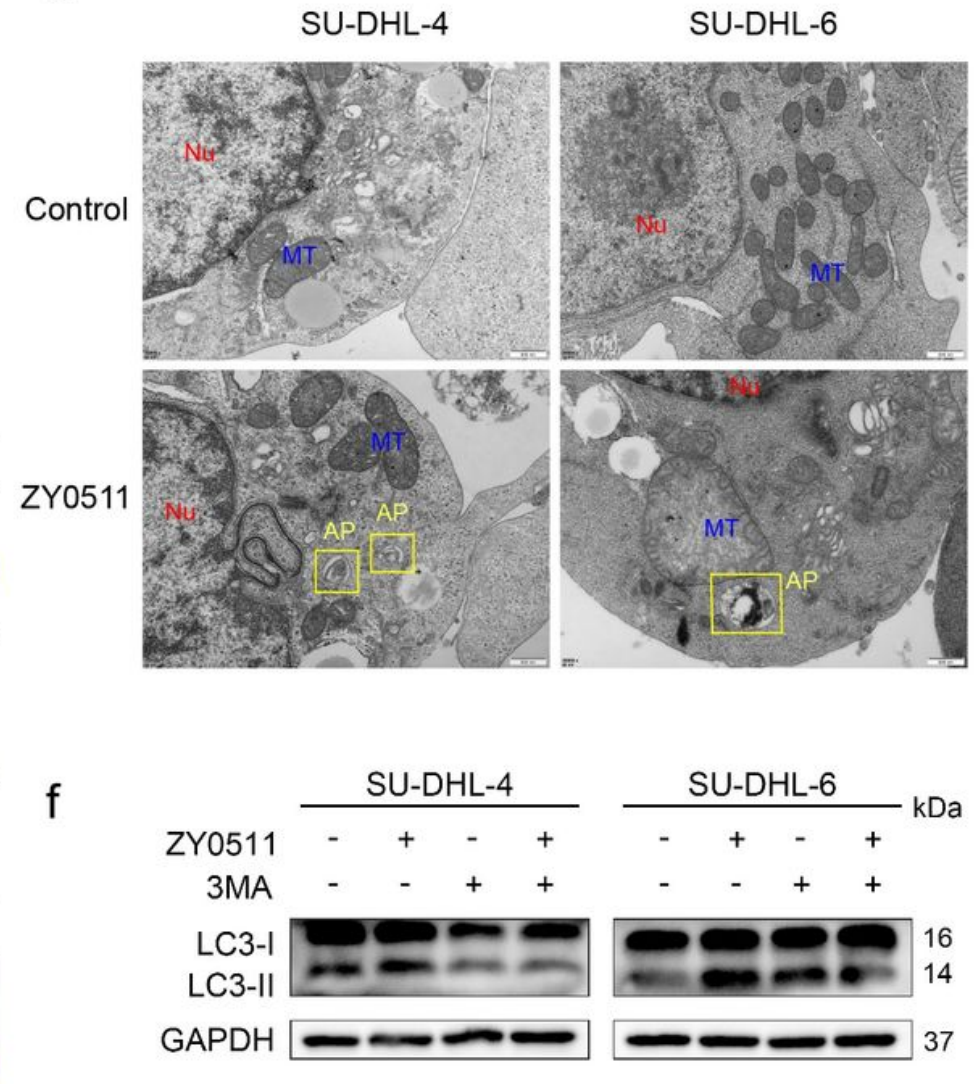

g

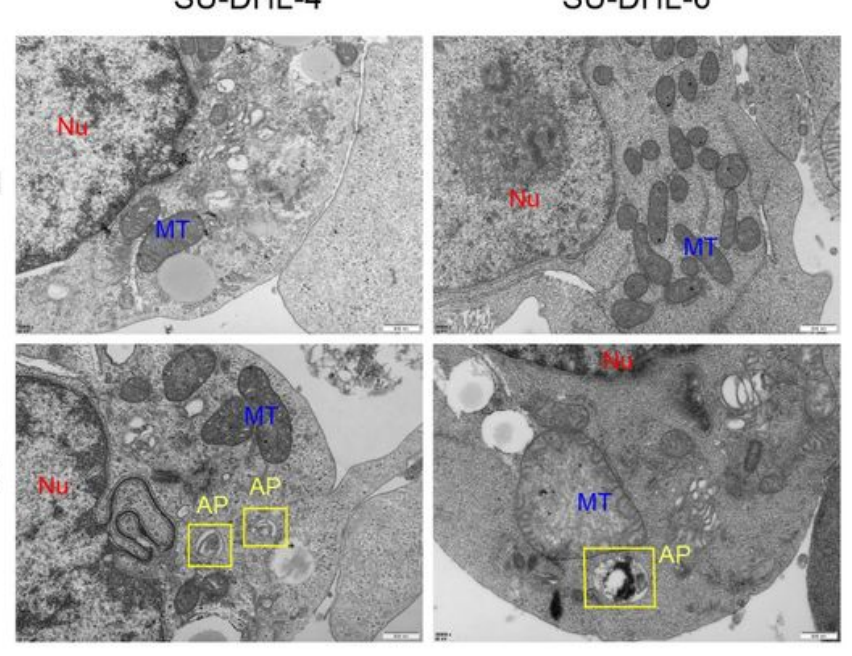

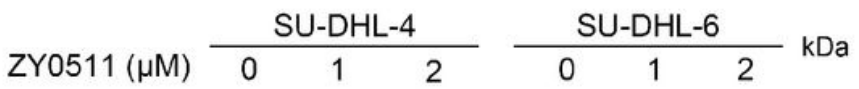
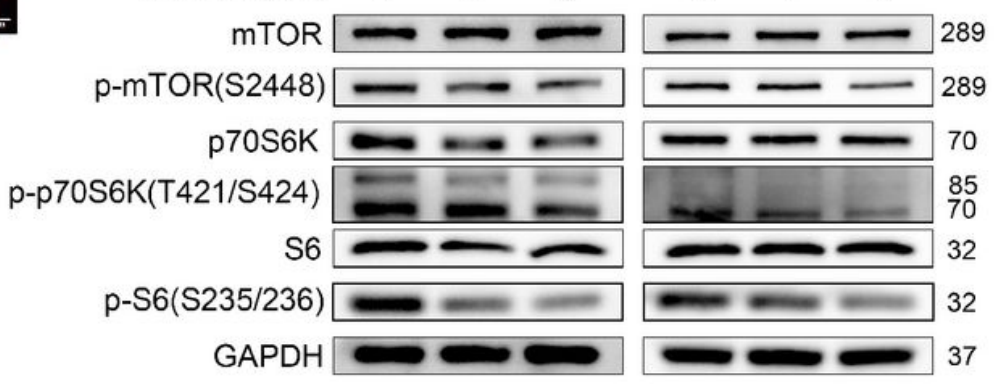

Figure 6 
ZY0511 induces autophagy in DLBCL Cells a Western blot detection of LC3B, p62, and Beclin-1 in DLBCL cells after ZY0511 treatment. b Representative images of LC3B detected by immunofluorescence, Nuclei are labeled with DAPI. Scale bar, $20 \mu \mathrm{m}$. c Representative images of p62 detected by immunofluorescence, Nuclei are labeled with DAPI. Scale bar, $20 \mu \mathrm{m}$. d Representative TEM images of autophagosomes in DLBCL cells with ZY0511 treatment. AP, autophagosome (yellow square); $M$, mitochondria; Nu, nucleus. Scale bar, $500 \mathrm{~nm}$. e Western blot detection of LC3B with ZY0511 and 3MA treatment. $f$ Western blot detection of LC3B and p62 with ZY0511 and CQ treatment. $g$ Western blot detection of the mTOR/p70S6K/S6 signaling pathway related proteins 